\title{
Croissance et régénération des racines de semis de pins laricio et de pins noirs en chambre climatisée et in situ
}

\author{
A. RIEDACKER *, M. ARBEZ * *
}

avec la collaboration technique de J.F. Mulder*, M. FAucher ** et de E. Henrion*:**

*I.N.R.A., Station de Sylviculture et de Production

Centre de Recherches forestières, Champenoux, F 54280 Seichanps

** I.N.R.A., Laboratoire d'Amélioration des Arbres forestiers

Domaine de L'Hermitage, Pierroton, F 33610 Cestas Principal

***: Dessinateur au C.N.R.F.

\begin{abstract}
Résumé
Nous avons observé la croissance aćrienne et souterraine de jeunes pins noirs d’Autriche (Pinus nigra ssp nigricans. Host.) et de jeunes pins Laricio de Corse (Pinus nigra ssp...) :

- d'une part, élevés en minirhizotrons en chambre climatisée pendant environ 6 mois, ou in situ (les racines étant visibles sur la paroi transparente d'une cave) pendant 2 ans:

- d'autre part, arrachés chaque mois, et repiqués soit en pépinière, soit en chambre climatisée (en minirhizotron).

Quatre phases de croissance racinaire ont été mises en évidence : une phase I qui précède le débourrement à croissance souterraine potentiellement forte, mais dont l'expression in situ est généralement linitée par la température; une phase II à faible potentialité de croissance souterraine qui se situe pendant la période d'élongation de la pousse terminale; une longue phase III à forte potentialité de croissance qui débute après la fin de l'élongation de la pousse terminale, et une phase IV à faible potentialité de croissance souterraine qui se situe vers la fin de l'été. La vitesse de déroulement de ces phases dépend de la température ambiante et l'expression des potentialités de croissance et de régénération est modulée par la température du sol. In situ, les basses températures hivernales peuvent interrompre toute croissance racinaire.
\end{abstract}

A l'intérieur des phases de croissance s'inscrivent des vagues de croissance espacées de 2 à 4 semaines. La présence de pointes racinaires blanches, même peu nombreuses, semble pouvoir aider les plants à survivre quelque temps, souvent jusqu'à ce qu'une vague de régénération des racines suffisamment forte apparaisse. Une interprétation des notions de potentiel de régénération des racines est donnée à la suite des observations qui ont pu être faites.

D'un point de vue pratique, les repiquages en pépinière en fin d'été, au début de la période III, débutant immédiatement après la fin de l'élongation de la pousse terminale, permettent la meilleure élongation de la pousse terminale au cours de l'année suivante. Les repiquages pendant la période II, en particulier en mai, la dépriment en revanche fortement. Les soulevages en début de période III devraient entrainer la plus faible dépression de la croissance aérienne. 
La plantation de fin d'été à l'aide de plants en mottes devrait permettre d'obtenir la meilleure colonisation du sol et le choc de transplantation le plus faible. Compte tenu de l'effet de la température de l'air sur le déroulement des différentes périodes de croissance, les reboiscments de printemps et d'automne, avec des plants à racines nues devraient sans doute être réalisés avec des plants provenant de pépinières bénéficiant de climats différents.

\section{Introduction}

Les jeunes plants de pins laricio de Corse ont un taux de reprise après plantation très variable, ce qui en limitc l'utilisation dans les reboisements. D'autres sousespèces du pin noir, notamment les pins noirs d'Autriche, ont par contre une reprise réputée moins aléatoire. Cette étude a donc été entreprise pour mieux comprendre le déterminisme de la croissance et de la régénération des racines de ces jcunes pins.

Nous avons pour cela étudié et comparé :

- d'une part la croissance aérienne et souterraine de plants placés durant une longue période soit en chambre climatisée, soit in situ;

- dautre part la régénération de racines et la survie de plants repiqués de manière échelonnée, soit en chambre climatisée, soit in situ.

\section{Matériel et méthode}

\subsection{Etude en chambre climatisie}

Des pins noirs d'Autriche (Pinus nigra ssp nigricans Host.) (abrégé : P.N.A.) et des pins laricio de Corse (Pinus nigra ssp laricio Poir.) (abrégé : P.L.C.), sans autre précision de provenance, ont été semés au printemps 1973 à la pépinière de Champenoux, près de Nancy, et prélevés à partir de l'automne 1974 pour les études en chambre climatisée.

Chaque mois, 6 plants de chaque sous-espèce ont été prélevés en pépinière. Leur système racinaire a été coupé à $20 \mathrm{~cm}$ du collet et toutes les pointes blanches sur la partie conservée ont été coupées à l'aide d'une paire de ciseaux (Série I).

Chaque mois, 6 autres plants de chaque sous-espèce ont également été prélevés, mais en essayant de garder les pointes blanches intactes (Série II). Cela ne fut pas toujours possible et seuls les plants dont les pointes blanches sallongèrent de quelques. millimètres furent conservés dans cette seconde série. En hiver, et en particulier en décembre et janvier, aucun plant ne présentait de pointes blanches. En août, les pointes blanches ne s'allongèrent généralement pas, malgré les précautions prises.

Ces plants ont ensuite été placés sur tourbe en minirhizotrons de $30 \mathrm{~cm} \times 70 \mathrm{~cm}$ (RiEdacker, 1974), puis transférés en chambre climatisée $\left(20 \pm 1{ }^{\circ} \mathrm{C}\right.$, jour et nuit - 2500 à 3000 Lux obtenus par tubes Sylvania Grolux - 16 heures de lumière - Humidité : 70 à 85 p. 100). 
A la mise en place, la tourbe a été saturée par une solution nutritive diluée (Coïc, 1973) et chaque semaine, au moment de la lecture de la croissance des racines, les minirhizotrons ont été retrempés durant environ $5 \mathrm{mn}$ dans cette solution à température de la chambre climatisée, afin de resaturer la tourbe.

Chaque semaine, nous avons noté : sique) ;

- la dessication des plants (au toucher et par perte des aiguilles au contact phy-

- le débourrement (ouverture des bourgeons, avec sortie des aiguilles);

- l'allongement de la pousse terminale et de ses aiguilles mesuré au double décimètre ;

- I’allongement, la ramification et la régénération des racines sur calque transparent, à l'aide de crayons marqueurs de couleur indélébile (une couleur différente par semaine). L'allongement hebdomadaire des pointes d'un système racinaire a été obtenu par lecture des relevés, à l'aide d'un curvimètre (précision de l'ordre du $\mathrm{mm}$ ).

Selon les cas, les plants ont été observés pendant 2 à 3 mois, ou 7 à 8 mois, tant que la lecture de la croissance des racines paraissait possible de manière satisfaisante.

\subsection{Etude des plants in situ}

La croissance des racines de 8 pins laricio de Corse (provenance Vezzani) de 3 ans a été suivie pendant deux ans, en 1968 et en 1969. Ces pins ( 1 an de semis et 2 ans de repiquage) avaient été repiqués en novembre 1967 derrière une plaque vitrée d'une chambre d'observations souterraines, dans un mélange de terre franche argilolimoneuse $(2 / 3)$ et de terre de bruyère $(1 / 3)$.

Le dispositif expérimental, ainsi que certains résultats relatifs à cette étude, ont déjà été décrits précédemment (ARBEZ, 1971). Dans la présente note, nous avons cependant transformé la présentation des données, notamment pour micux permettre la comparaison des résultats obtenus in situ avec ceux obtenus en chambre climatisée. A la place de la vitesse moyenne d'allongement des racines, nous avons préféré comme indice de croissance la somme des allongements hebdomadaires de l'ensemble des pointes visibles d'un système racinaire : c'est la vitesse d'extension d'un système racinaire.

Pour la partie aérienne, c'est l'élongation hebdomadaire de la pousse terminale qui a été mesurée au moyen d'une toise (précision de l'ordre de $2 \mathrm{~mm}$ ).

Le milieu a été caractérisé par les paramètres suivants : écoulée) ;

- températures de l'air (moyennes des minimas ou des maximas de la semaine

- température du sol et $\mathrm{pF}$ à $10,20,40$ et $80 \mathrm{~cm}$ de profondeur.

Pour étudier la régénération des racines in situ, nous avons en outre repiqué chaque mois, sauf en décembre, janvier et février, du 15 août 1970 au 15 août 1971 des semis d'avril 1970 effectués sur un lit de $30 \mathrm{~cm}$ de terre de bruyère.

Après arrachage, les extrémités blanches de chaque semis (supérieures à $3 \mathrm{~mm}$ ) ont été dénombrées, puis supprimées au sécateur. Les semis ont ensuite été mis en jauge pendant 4 semaines, sous ombrière haute, dans de la terre de bruyère humide. 
A l'issue de cette période de mise en jauge, les extrémités blanches de plus de $3 \mathrm{~mm}$ nouvellement apparues ou reconstituées ont été dénombrées et la longueur de la partie aérienne a été mesurée.

Toutes ces manipulations et observations des racines eurent lieu très rapidement à l'abri du soleil et du vent.

Les semis furent enfin repiqués à $12,5 \times 12,5 \mathrm{~cm}$ d'espacement, en bâche de terre franche, dans les conditions normales de la pépinière, suivant un dispositif en blocs complets à trois répétitions et 16 individus par combinaison provenance $X$ date de repiquage.

A l'automne 1972, soit un an après le dernier repiquage, la survie, la hauteur totale et la pousse annuelle de chaque individu furent enregistrées.

La sous-espèce laricio était représentée par deux provenances, l'une de Corse et l'autre de Calabre:

- VALD II, forêt domaniale de Valdoniello, France (Corse), alt. $1100 \mathrm{~m}$;

- GRAN, Grancia, forêt domaniale de Baraccone (Sila Greca), Italie (Calabre), alt. $850 \mathrm{~m}$; la sous-espèce nigricans était représentée par une seule provenance originaire de Bosnie :

VGRA I, forêt de Visegrad, Yougoslavie (Bosnie), altitude non précisée.

Au repiquage, la partie aérienne atteignait une hauteur moyenne respectivement de $7,2 \mathrm{~cm}, 6,2 \mathrm{~cm}$ et $5,4 \mathrm{~cm}$ pour les pins de Calabre, les pins noirs d'Autriche et les pins laricio de Corse.

\subsection{Présentation des résultats}

Nous avons cherché à présenter les résultats sous forme de diagrammes de croissance, où figurent simultanément les croissances aériennes et souterraines. Cela fut possible pour les différents traitements, sauf pour celui relatif à l'étude de la régénération des racines de plants repiqués en jauge.

Pour les observations de longue durée, nous avons en outre fait figurer pour la partie souterraine les courbes lissées et les courbes des écarts entre la valeur brute et la valeur lissée. Le lissage des courbes a été obtenu par application de la formule suivante :

$$
\bar{x}_{1}=\frac{x_{i-1}+2 x_{i}+x_{i+1}}{4}
$$

Il permet de séparer des tendances fondamentales du phénomène observé des petites fluctuations qui pourraient être dues soit à des variations régulières autour de la courbe lissée, soit à des erreurs.

Nous avons présenté à la fois des diagrammes relatifs à un plant donné, et des diagrammes moyens. Ces derniers font perdre certaines informations. Ils permettent cependant de visualiser la généralité des phénomènes et de montrer parfois certaines synchronisations. 
Enfin, étant donné que de nombreux auteurs utilisent les notions de potentiel de régénération des racines (RitchiE \& DunlaP, 1980), nous avons également utilisé ce critère. Sur des systèmes racinaires sectionnés à $20 \mathrm{~cm}$ du collet, privés de toutes leurs pointes blanches et placés en chambre climatisée à $20^{\circ} \mathrm{C}$, c'est-à-dire à des températures favorables à la croissance et à la régénération des racines, nous avons compté le nombre moyen de pointes blanches apparues au cours des 3 semaines qui ont suivi la transplantation.

\section{Résultats}

\subsection{Analyse des diagrammes de croissance de plants} conservés en chambre climatisée pendant 6 mois (fig. 1)

La croissance des pins laricio de Corse transférés de la pépinière en chambre climatisée le 4 décembre a été observée jusqu'en mai. Dans ces conditions, nous remarquons que la pousse terminale ne s'allonge pas. Les aiguilles, en revanche, s'allongent assez régulièrement après le débourrement qui se produit entre le 24 décembre et le 9 janvier pour tous les plants. Un second débourrement de bourgeons latéraux a lieu début mai sur 2 plants, dont celui présenté en fig. $1 \mathrm{~A}$.

Le système racinaire présente deux périodes de croissance forte (phases I et III), alternant avec des phases de croissance quasiment nulle (phases II et IV). La phase I est relativement courte et précède le débourrement, alors que la phase III s'étale du début février au début avril. La phase IV est également plus longue que la phase II.

Lorsque les pins sont transférés de la pépinière à la chambre climatisée le 14 mars avec leurs pointes blanches, l'allure générale du diagramme (fig. 1 B) est comparable au précédent. Le débourrement a lieu fin mars/début avril. Dès le 18 juin, les nouveaux bourgeons terminaux sont bien visibles. Ils se gonflent, puis, le 20 août, trois bourgeons débourrent sur un des plants, tandis que le 3 septembre, 6 bourgeons débourrent sur un autre plant. A cette date, les bourgeons des autres plants du lot sont bien verts et prêts à débourrer. On remarque cependant qu'à partir du 20 août, avant le débourrement, une seconde période de croissance forte débute. Celle-ci est visible sur tous les plants, sauf sur le plant qui a débourré le plus précocement, c'est-à-dire le 20 août.

A ces phases de croissance globalement fortes et faibles, se superposent d'autres fluctuations. Toutes les 2,3 ou 4 semaines, apparaissent des pics de croissance (fig. 1 et 2), ainsi que des pics de ramification des racines (fig. 2). Ces fluctuations sont relativement bien marquées sur les différentes courbes individuelles et moyennes. C'est toutefois durant la phase III, c'est-à-dire durant la longue période de croissance racinaire globalement forte, qu'elles sont le mieux marquées (Vagues $n^{\circ} 3$, 4 et 5).

On les retrouve aussi bien sur les courbes indiquant le nombre de nouvelles racines apparues depuis la précédente semaine que sur la somme des allongements racinaires. Elles sont moins nettement visibles sur la courbe représentant le nombre de racines en croissance. 


\subsection{Analyse des diagrammes de croissance des plants en pépinière}

Sur la fig. 3, nous avons représenté les diagrammes lissés de la vitesse moyenne d'extension racinaire de pins laricio de Corse en 1968 et 1969.

Il apparaît nettement 5 phases; trois phases ont des croissances racinaires relativement fortes en mai (I et 1), en juillet-août (III et 3), et en automne (Ib et 1b) ; et deux phases, en juin (II et 2) et à la fin de l'été (IV et 4), présentent des croissances racinaires relativement faibles. On remarquera que si les deux premières phases sont relativement bien synchronisées au printemps, les phases suivantes sont décalées. La phase II dure plus longtemps et paraît moins accentuée en 1969. Elle se termine vers la mi-juillet au lieu de la fin juin.

FIG. 1

Diagramme de croissance de pins laricio transférés de la pépinière en chambre climatisée $\left(20^{\circ} \mathrm{C}-16 \mathrm{~h}\right.$ de lumière $\left.-3000 \mathrm{Lux}\right)$.

A : Diagramme d'un plant transféré le 4 décembre.

$B$ : Diagramme d'un plant transféré le 14 mars.

Growth diagrams of Corsican Pine seedlings transfered from the nursery into the growth chamber

$\left(20^{\circ} \mathrm{C}-16 \mathrm{~h}\right.$ dayligth $\left.-3000 \mathrm{Lux}\right)$.

A : Diagram of a seedling transfered on 4th December.

$B$ : Diagram of a seedling transfered on 14th March.

- Partie aérienne :

- Trait fort : Longueur des aiguilles de la pousse terminale.

- Trait fin : Allongement hebdomadaire des aiguilles de la pousse terminale.

- Cercles : Nombre de bourgeons qui débourrent.

- Partie souterraine :

- Trait fort : Somme des allongements hebdomadaires des racines visibles sur le minirhizotron.

- Tireté vertical : Pics des vagues de croissance :

I et III : phases de forte croissance racinaire;

II et IV : phases de faible croissance racinaire.

- Aerial part:

- Thick line: Length of the needles of the terminal shoot.

- Thin line: Weekly elongation of the needles of the terminal shoot.

- Circles : Number of budbreakings.

- Subteranean part :

- Thick line : Weekly extension growth of roots visible on the transparent side of the minirhizotron.

- Vertical broken lines : peaks of growth waves:

I and III : Periods of strong root growth;

$I I$ and IV : Periods of weak root growth. 

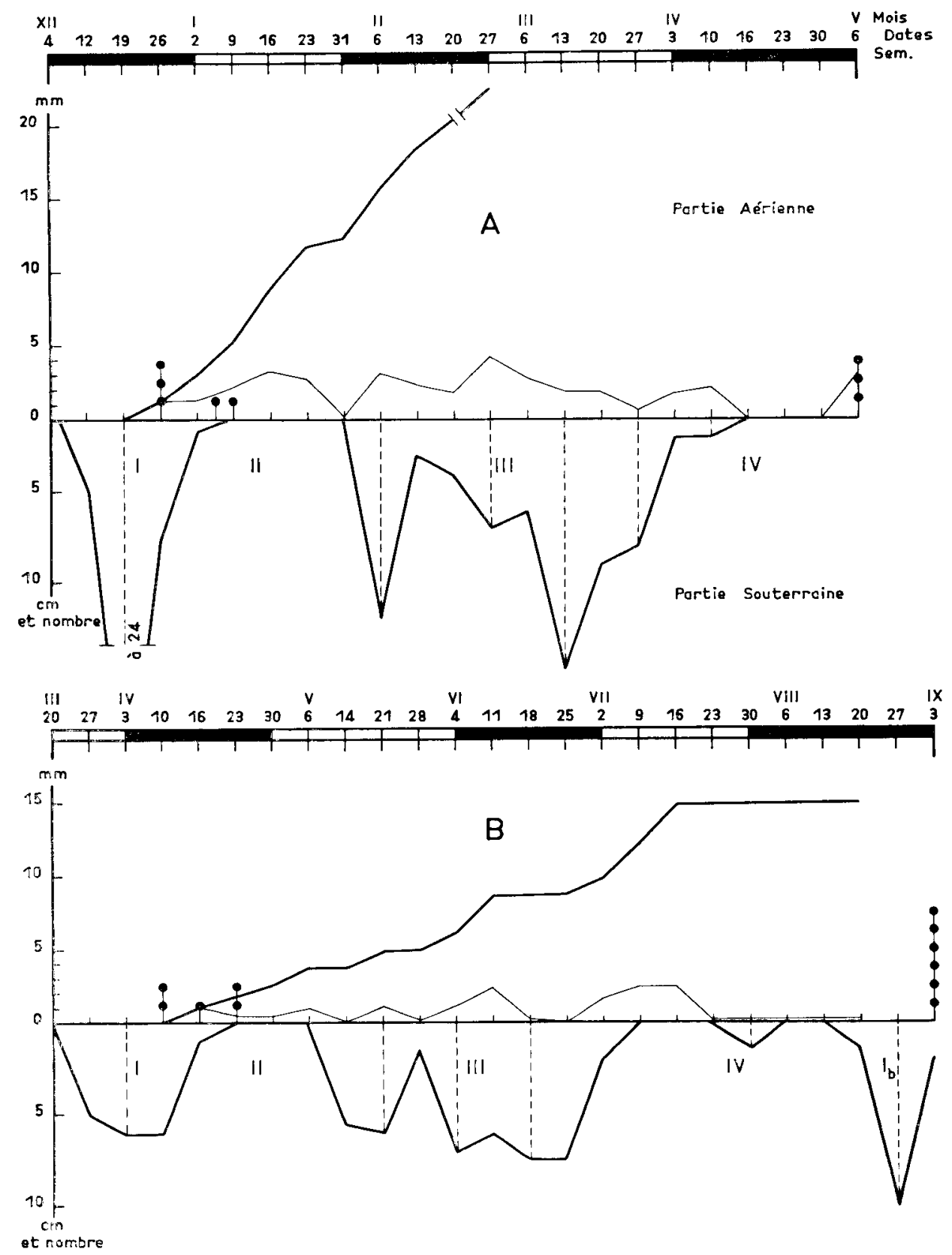

FIG. 1 
En examinant la croissance des pousses terminales, on s`aperçoit qu'en 1968 la vitesse d'élongation maximale en juin est en moyenne trois fois plus élevée qu'en 1969. On s’aperçoit aussi que la croissance est plus étalée dans le temps. Lorsqu'on ne considère que la première pousse, elle dure du 18 avril au 13 juin, soit 8 semaines en 1968, et du 3 avril au 17 juillet, soit 15 semaines en 1969.

Les températures du printemps 1969 ont été plus fraîches que durant le printemps 1968. En 1968, c'est en particulier vers la fin juin que l'on note les températures les plus élevées, alors qu’en 1969, des températures comparables ne sont atteintes qu'en juillet. Ces différences de températures qui déterminent aussi directement la vitesse de croissance des pousses aériennes pourraient bien expliquer les décalages observés au niveau souterrain.

Les débuts des phases III et IV sont en effet retardés en 1969 d'une quinzaine de jours. La phase IV, au lieu d'apparaître en septembre, apparaît début octobre, et la phase 1b, qui était bien visible en 1968, est très courte et peu distincte en 1969 (Ib).

FIG. 2

Diagrammes moyens de la croissance souterraine de 6 pins laricio transférés en chambre climatisée le 14 mars.

Mean diagrams of root of 6 Corsican Pine seedlings transfered into the gronth chamber on March 14.

B : Courbes brutes.

L : Courbes lissées.

$\mathbf{E}_{1}$ : Courbe des écarts entre la valeur brute et la valeur lissée du nombre de nouvelles ramifications visibles.

$E_{2}$ : Courbe des écarts entre les valeurs brutes et les valeurs lissées de la somme hebclomadaire des allongements et du nombre total de racines visibles en croissance.

- Trait fort : Moyenne des allongements hebdomadaires des racines visibles sur le minirhizotron.

- Trait fin : Nombre moyen de racines visibles et en croissance.

- Pointillé : Nombre de nouvelles ramifications apparues depuis une semaine.

- Pointillé vertical : Vagues de croissance.

$B$ : Original curves.

$L$ : Smoothed curves.

$E_{1}$ : Difference between the original and smoothed value for the number of visible ramifications.

$E_{z}$ : Difference between the original and smoothed value for the weekly extension growth of visible roots and the total number of growing roots.

- Thick line : Mean extension growth of visible roots.

- Thin line : Mean number of visible growing roots.

- Dotted line : Number of new ramifications per week.

- Vertical dotted line : Growth waves. 


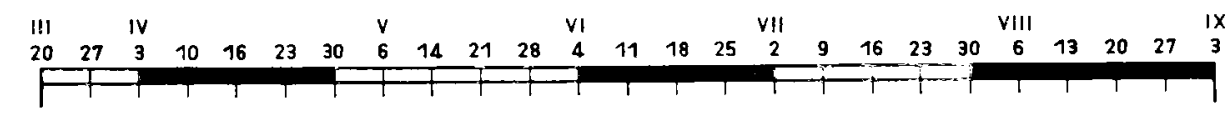
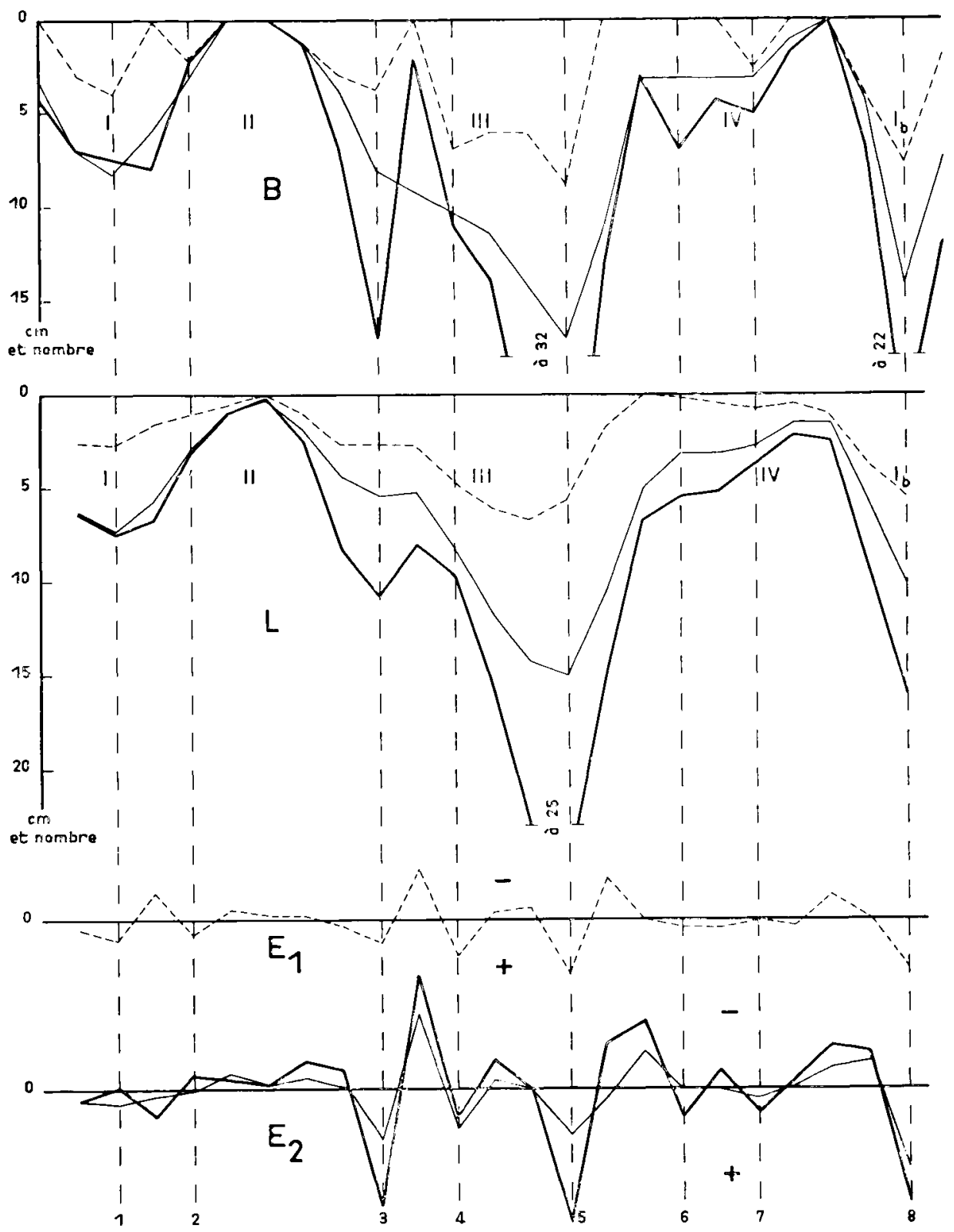

FIG. 2 


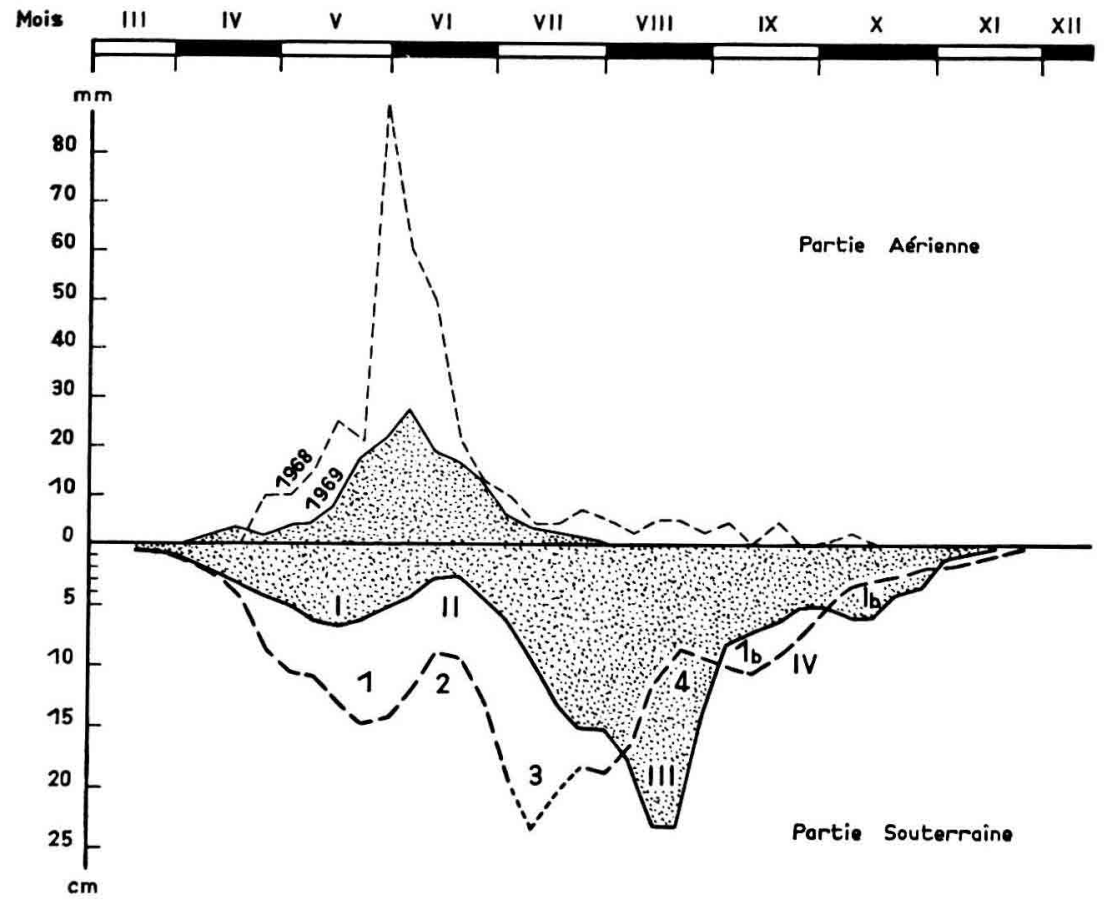

FIG. 3

Diagrammes lissés de vitesse moyenne d'extension racinaire de 8 pins laricio de Corse in situ.

Smoothed diagrams (the mean root extension speed) of 8 Corsican pines in situ.

$1-2-3-4-1 b$ : Phases en 1968.

I - II - III - IV - 1b : phases en 1969.

$1-2-3-4-1 b$ : periods in 1968.

I $-I I-I I I-I V-I b$ : periods in 1969.

FIG. 4

Relation entre la température du sol à $-10 \mathrm{~cm}$ et $-80 \mathrm{~cm}$ de profondeur et l'allongement moyen hebdomadaire lissé des racines en 1968 et en 1969.

Relationship between soil temperature (at $-10 \mathrm{~cm}$ and $-80 \mathrm{~cm}$ ) and the smoothed weekly extension growth of visible roots in 1968 and 1969.

Le gros point indique le début de la première semaine du mois repéré par un chiffre romain.

$\mathbf{R}=$ Courbe de régression tracée à main levée.

The full circle indicates the beginning of the first week of the month designated by a roman figure.

$R=$ Hand drawn regression line. 
Allongement hebdomadaire

moyen lissé en cm

(10

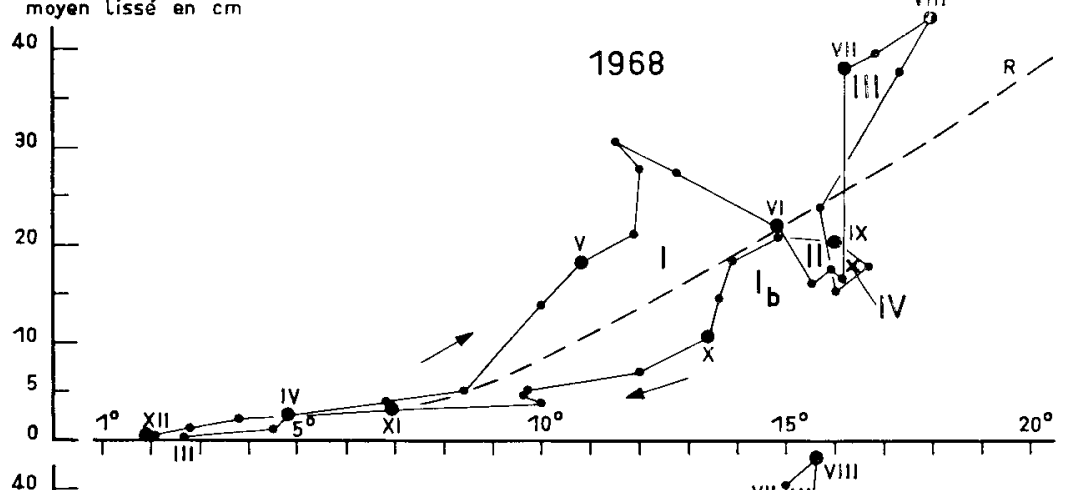

d $-10 \mathrm{~cm}$

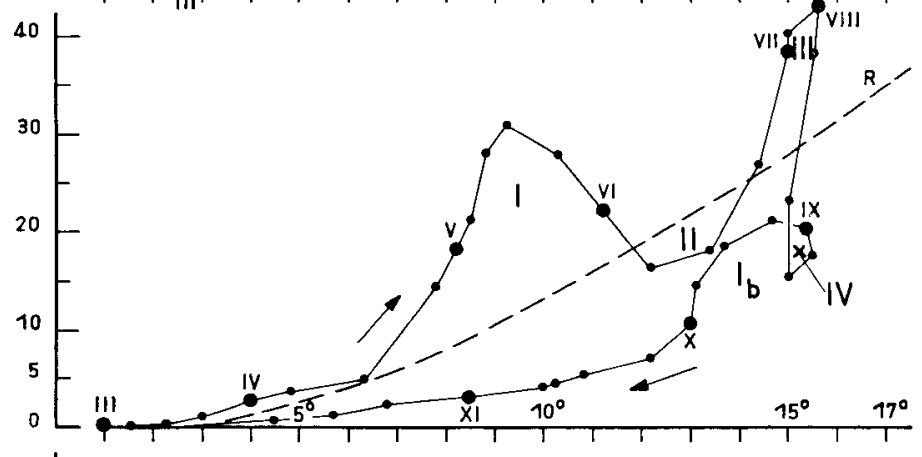

à $-80 \mathrm{~cm}$

40

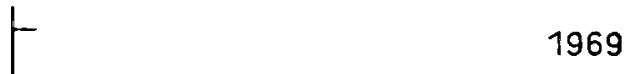

30

1969

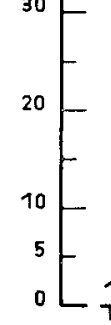

40
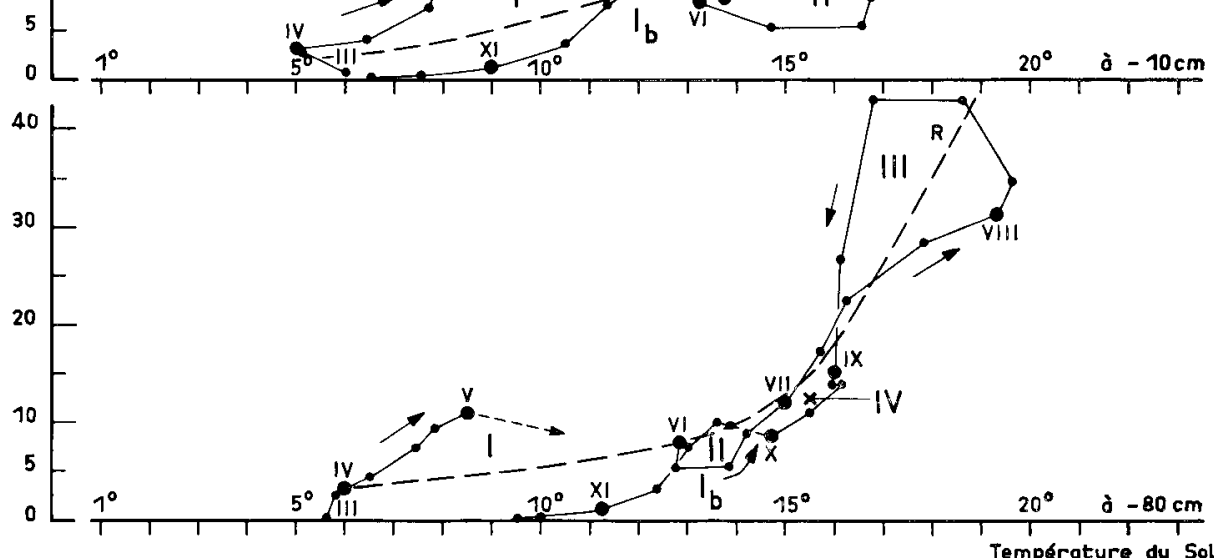

on ${ }^{\circ} \mathrm{C}$

FIG. 4 
Lorsqu'on représente les valeurs lissées des vitesses moyennes d’extension des systèmes racinaires, en fonction de la température du sol à $-10 \mathrm{~cm}$ ou à $-80 \mathrm{~cm}$ de profondeur (fig. 4), il apparaît nettement que cette vitesse d'extension augmente en movenne avec la température du sol (courbe de régression $\mathrm{R}$ ). Ce résultat est classique, mais lorsquion relie les points entre eux, les phases évoquées ci-dessus apparaissent encore plus clairement.

Toutes ces phases peuvent aussi être mises en évidence sur les diagrammes individuels (fig. 5). On remarquera alors que, sur le plant représenté, la croissance aérienne en 1969 est plus forte que la moyenne et que la phase II est plus accentuée que sur le diagramme moyen. Ce plant ne présente pas de phase Ib en 1969. Seulement 2 des 8 plants présentent cette phase, tandis que sur 2 autres plants, la phase III se proIongeait jusqu'en octobre. Ces petits décalages créent donc des artefacts sur la courbe moyenne, puisque la phase Ib est ainsi renforcée.

A ces différentes périodes se superposent comme en chambre climatiséc des pics de croissance racinaire espacés de 2 à 4 semaines qui sont particulièrement nets au cours des phases I, III et Ib à fortes potentialités de croissance racinaire. Les fluctuations du nombre de racines qui poussent et de la somme des allongements racinaires (fig. 5 et 6 ) indiquent d'ailleurs également bien les pics. Mais, e'est sans doute parce que lallongement des racines est thermodépendant que les fluctuations du second paramètre sont plus importantes durant la phase III et égales ou moins importantes durant les phases $I$ et $I b$. D'un plant à l'autre, les pics peuvent être décalés de 1 à 2 semaines. Mais, pendant la phase III, il s'effectue un certain regroupement. C'est ainsi, qu'en 1968, 6 plants sur 8 présentent simultanément le pic $\mathrm{n}^{\circ} 9$ au cours de la même semaine.

FrG. 5 et 5 bis

\begin{abstract}
Diagramme de croissance d'un pin laricio in situ en 1968 et 1969.
Growth diagram of an individual Corsican Pine seedling in 1968 and 1969.
\end{abstract}

B : Diagramme brut.

L : Diagramme lissé de la partie souterraine avec les différentes phases indiquées en chiffres romains.

E : Courbe des écarts entre les valeurs brutes et les valeurs lissées relatives à la partie souterraine.

- Partie souterraine :

- Trait fort : Allongement hebdomadaire des racines visibles.

- Trait fin : Nombre de racines visibles qui poussent.

- Pointillé vertical : Vagues de croissance.

B : Original diagram.

$L$ : Smoothed diagram (the different periods are indicated with roman letters).

$E$ : Difference between the original and smoothed values.

- Subteranean part :

- Thick line : Weekly extension growth of visible roots.

- Thin line : Number of visible growing roots.

- Dotted vertical line : Growth waves. 

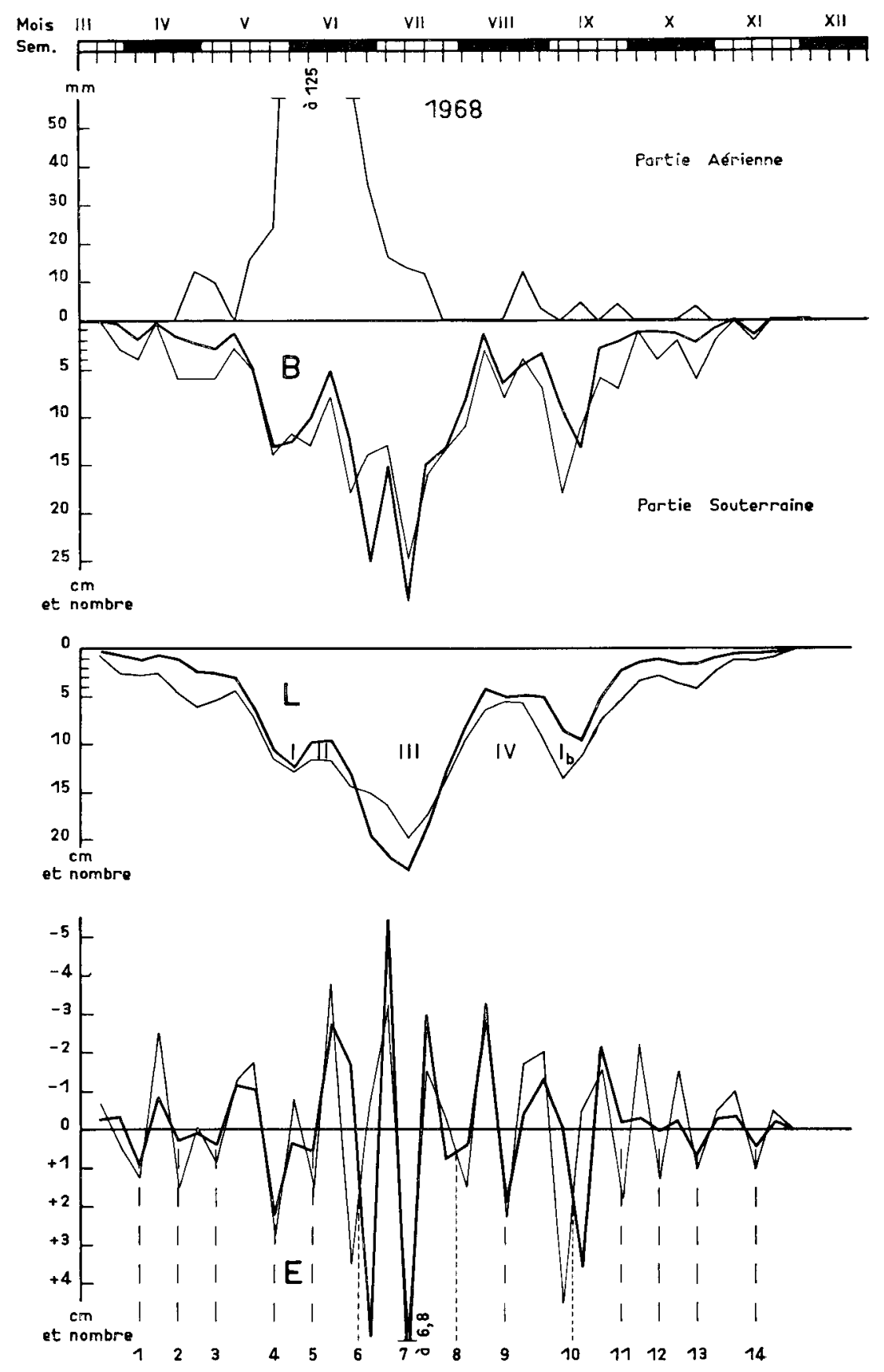

FIG. 5 
92

A. RIEDACKER, M. ARBEZ

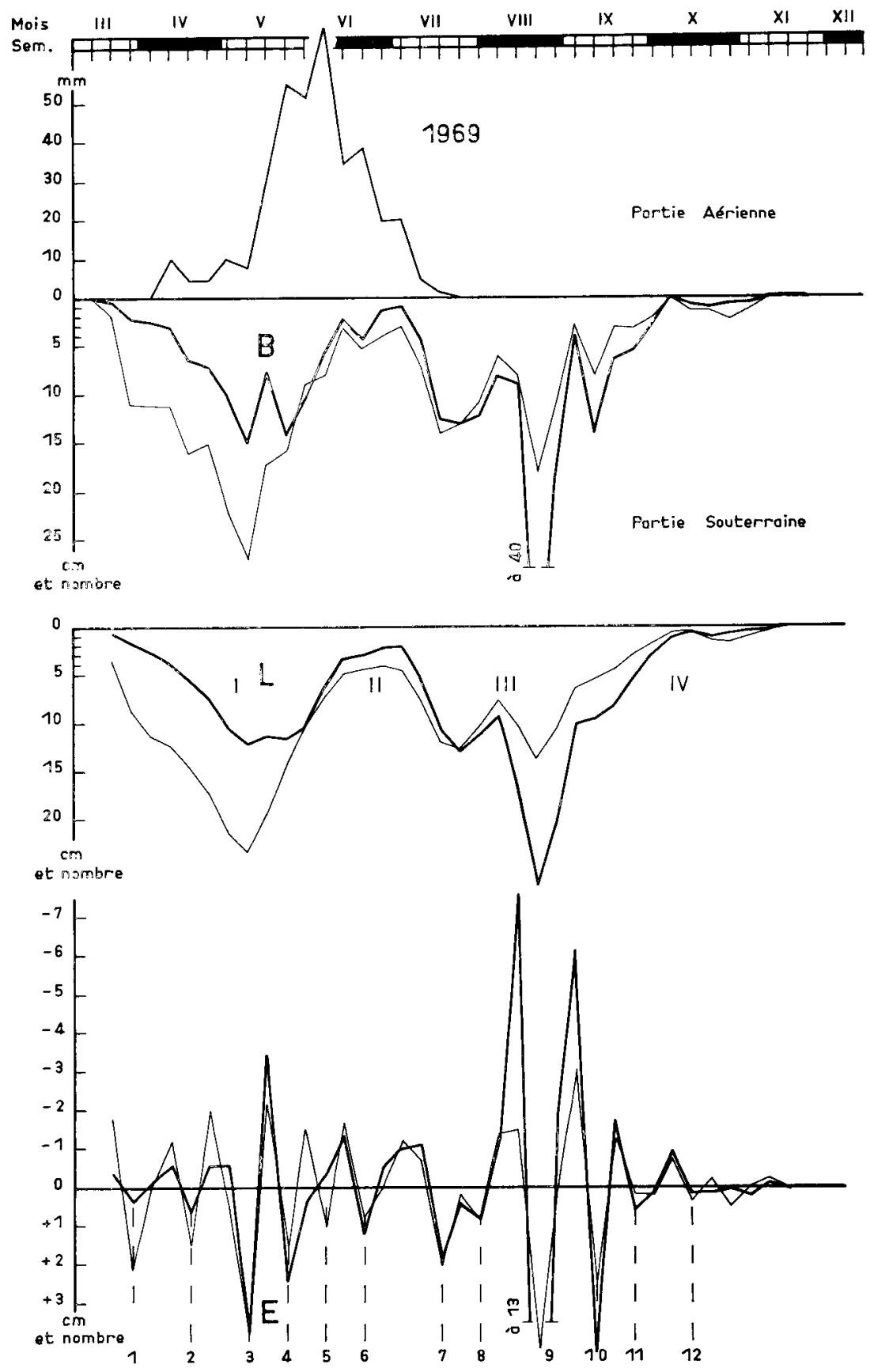

FIG. 5 bis 

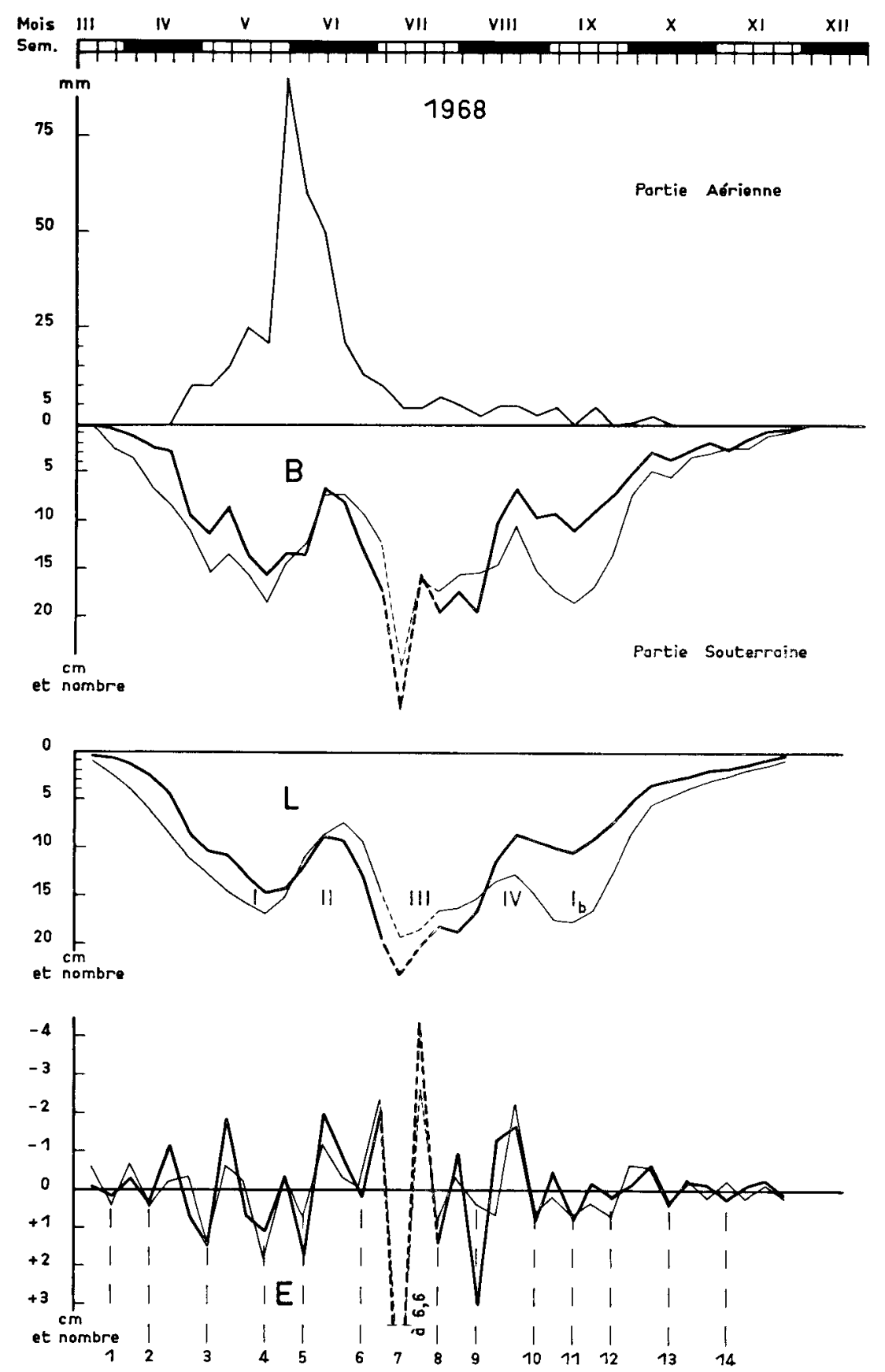

FIG. 6 


\subsection{Analyse des diagrammes de croissance de plants transférés en chambre climatisée de manière échelonnée tout au long de l'année}

Sur la fig. 7, nous présentons des diagrammes individuels de pin laricio de Corse pour chaque date de transfert en chambre climatisée. En novembre, on peut observer les phases I (croissance racinaire forte) et II (croissance racinaire faible) précédemment décrites.

En décembre et janvier, on peut en outre observer le début de la phase III (croissance racinaire forte) sur tous les plants.

Mais, en février, mars et avril, seulement un à deux plants sur six régénèrent des racines et survivent dans nos conditions. La phase I n'apparaît plus. Les plants entrent directement dans la phase II. Lors des transplantations du mois de mai, aucun plant ne survit.

En juin, la régénération des racines na lieu quaprès 3 semaines, lorsque les plants entrent dans la phase III. Sur ces plants, on peut aussi observer la phase IV (croissance racinaire faible) qui apparaît en septembre. Le seul plant transféré en chambre climatisée en avril et ayant survécu présente la première vague de croissance de la phase III, à peu près au même moment que les plants transférés en juin.

En juillet et août, aucun pin laricio de Corse, privé de ses pointes blanches, ne survit à la transplantation.

En octobre, seul un plant privé de ses pointes survit. Il présente une phase de croissance racinaire forte (phase Ib), suivie d'une croissance nulle, sans pourtant présenter de débourrement.

FII. 6 et 6 bis

Diagramme moyen de croissance de 8 pins laricio in situ en 1968 et 1969.

Mean growth diagram of 8 Corsican Pine seedlings grown outdoors in 1968 and 1969.

B : Diagramme brut.

L : Diagramme de la partie souterraine lissé avec les différentes phases indiquées en chiffres romains.

E : Courbe des écarts entre les valeurs brutes et les valeurs lissées relatives à la partie souterraine.

- Trait fort : Allongement moyen hebdomadaire des racines visibles.

- Trait fin : Nombre moyen de racines visibles qui poussent.

- Trait vertical en pointillé : Vagues de croissance.

(En pointillé : données manquantes, sauf pour un plant).

$B$ : Original diagram.

$L$ : Smoothed diagram with 4 periods indicated by roman numbers.

$E$ : Difference between the original and smoothed values.

- Thick line : Mean weekly extension growth of visible roots.

- Thin line : Mean number of visible growing roots.

- Dotted vertical line : Growth waves.

(Dotted lines : missing data). 
Mois 1 Sem. III
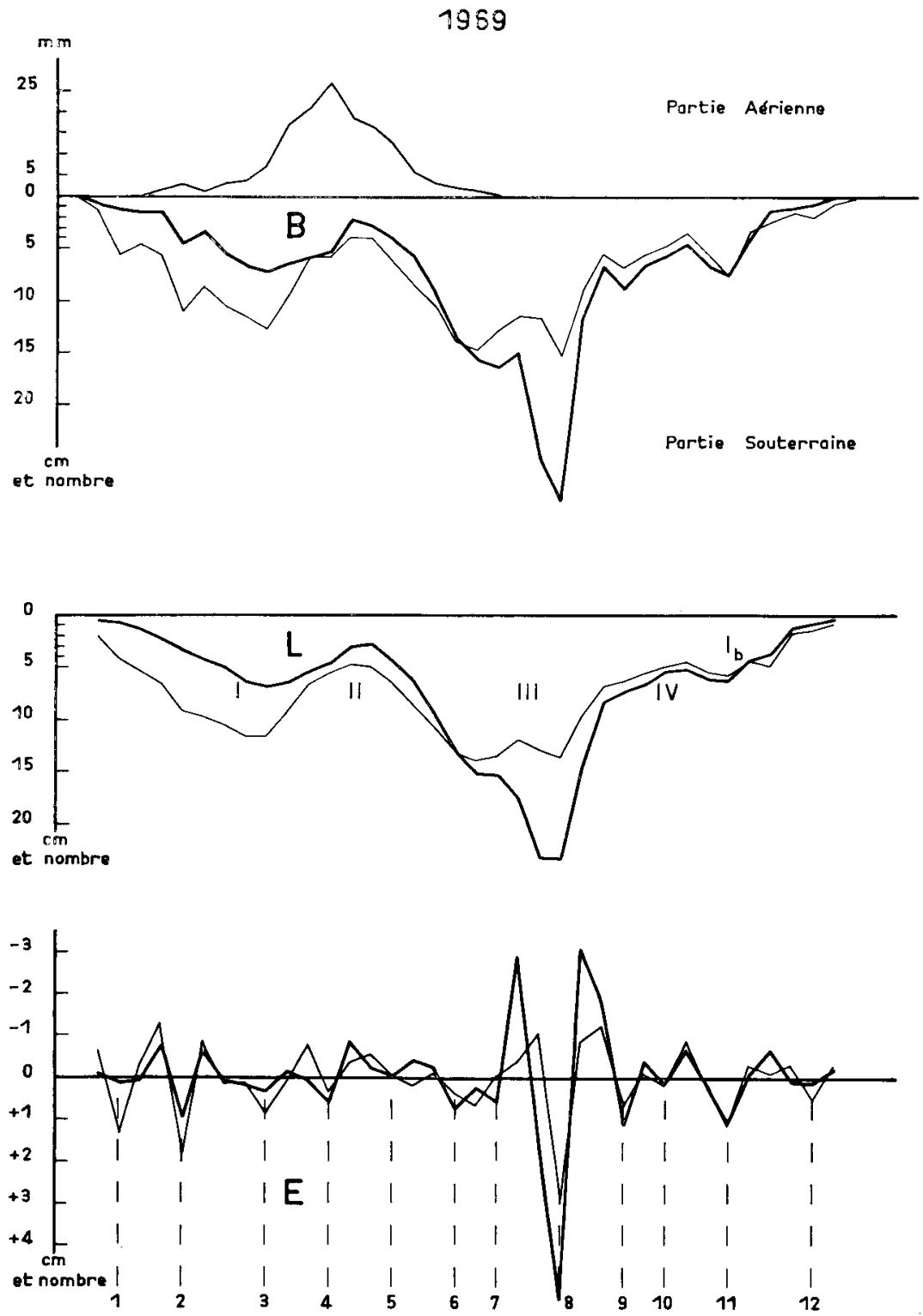

FIG. 6 bis 

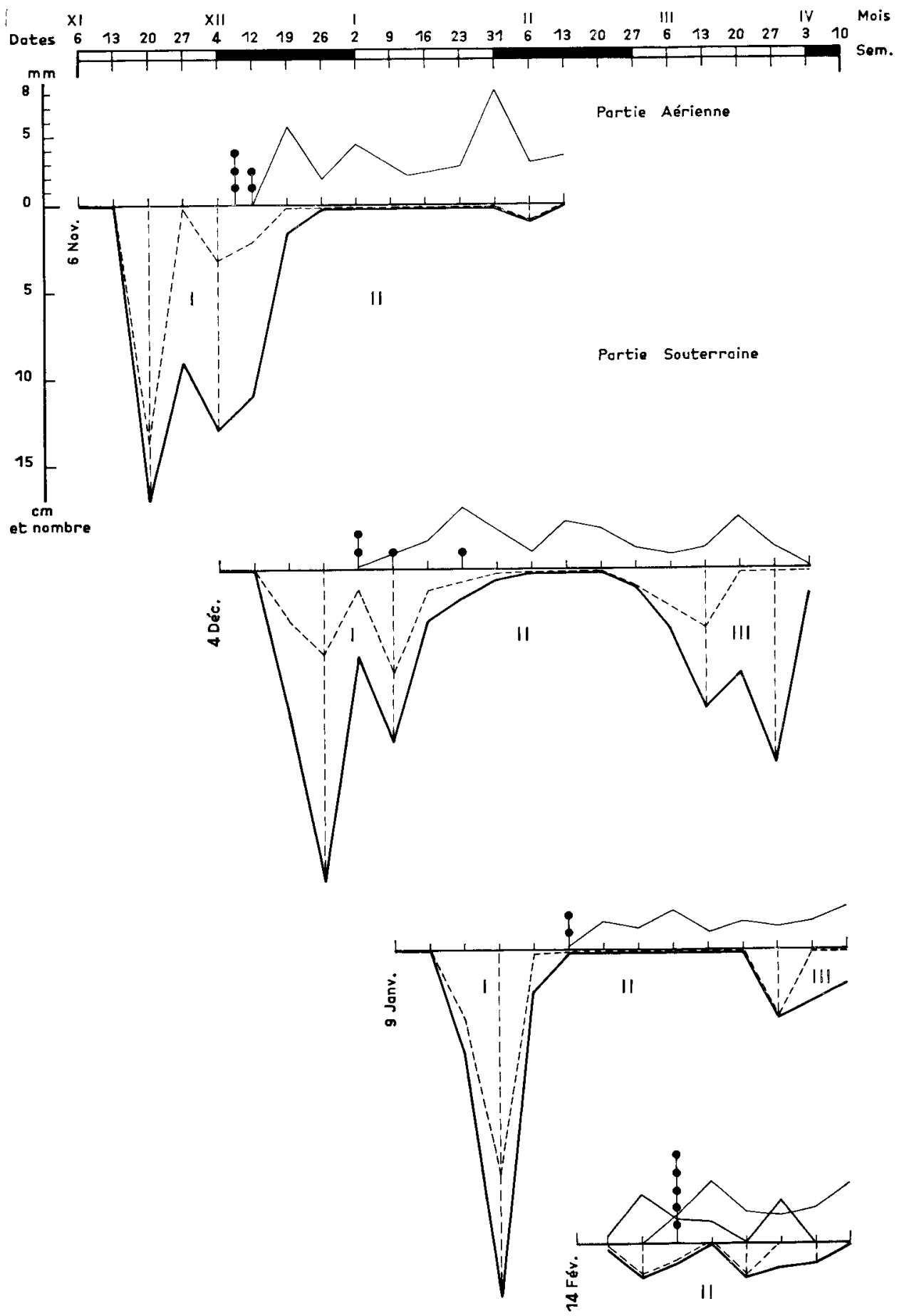

FIG. 7 


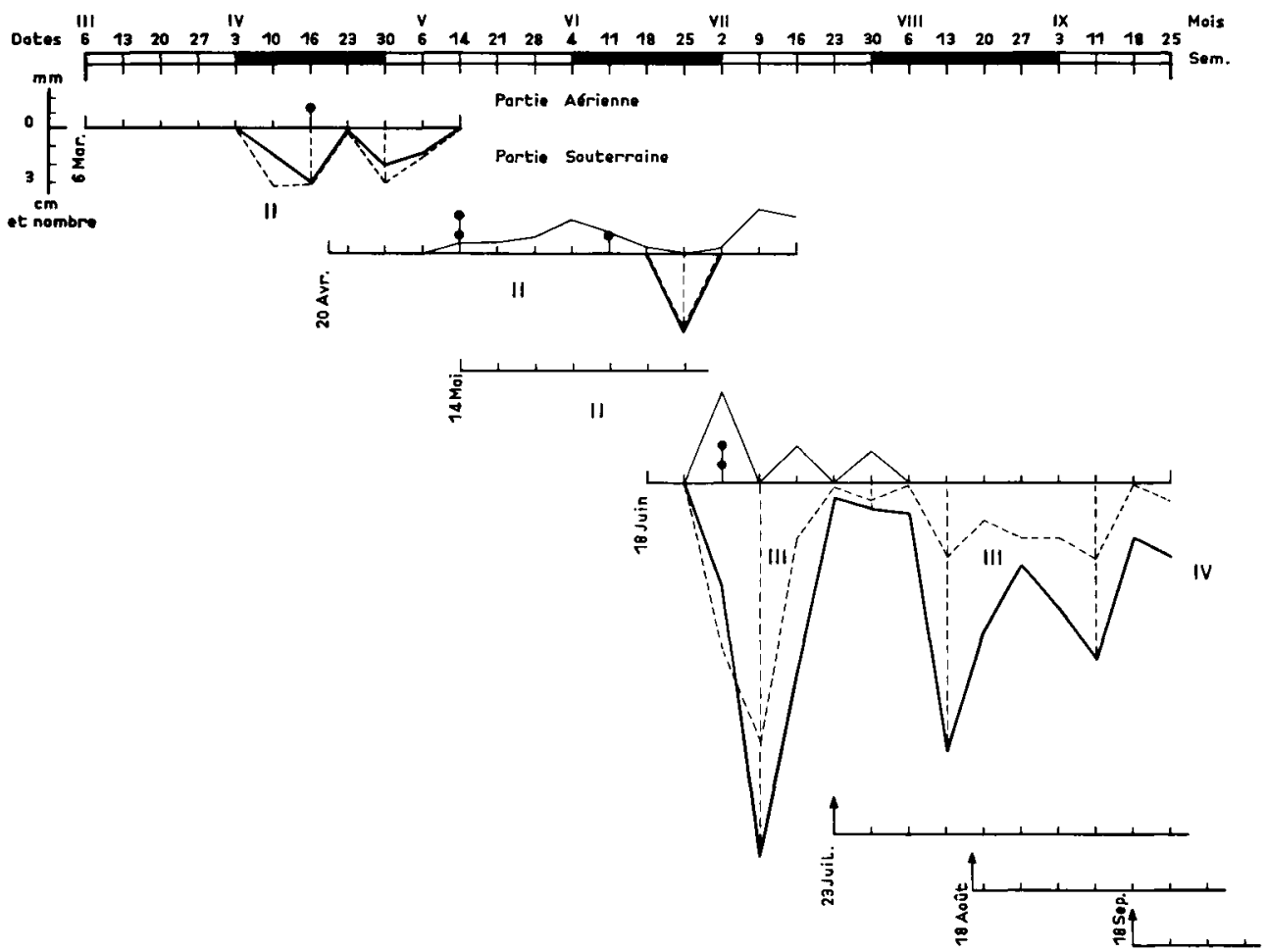

FIG. 7 bis

Diagrammes individuels de croissance de pins laricio de Corse transférés en chambre climatisée à différents moments de l'année.

Individual diagrams of Corsican Pine seedlings transfered from the nursery into the growth chamber at different periods.

- Les phases de croissance racinaire sont indiquées en chiffres romains.

- Les pics des vagues de croissance sont indiqués par les traits interrompus verticaux.

- Root growth periods are indicated with roman figures.

- Peaks of root gronth wates are indicated by broken vertical lines. 

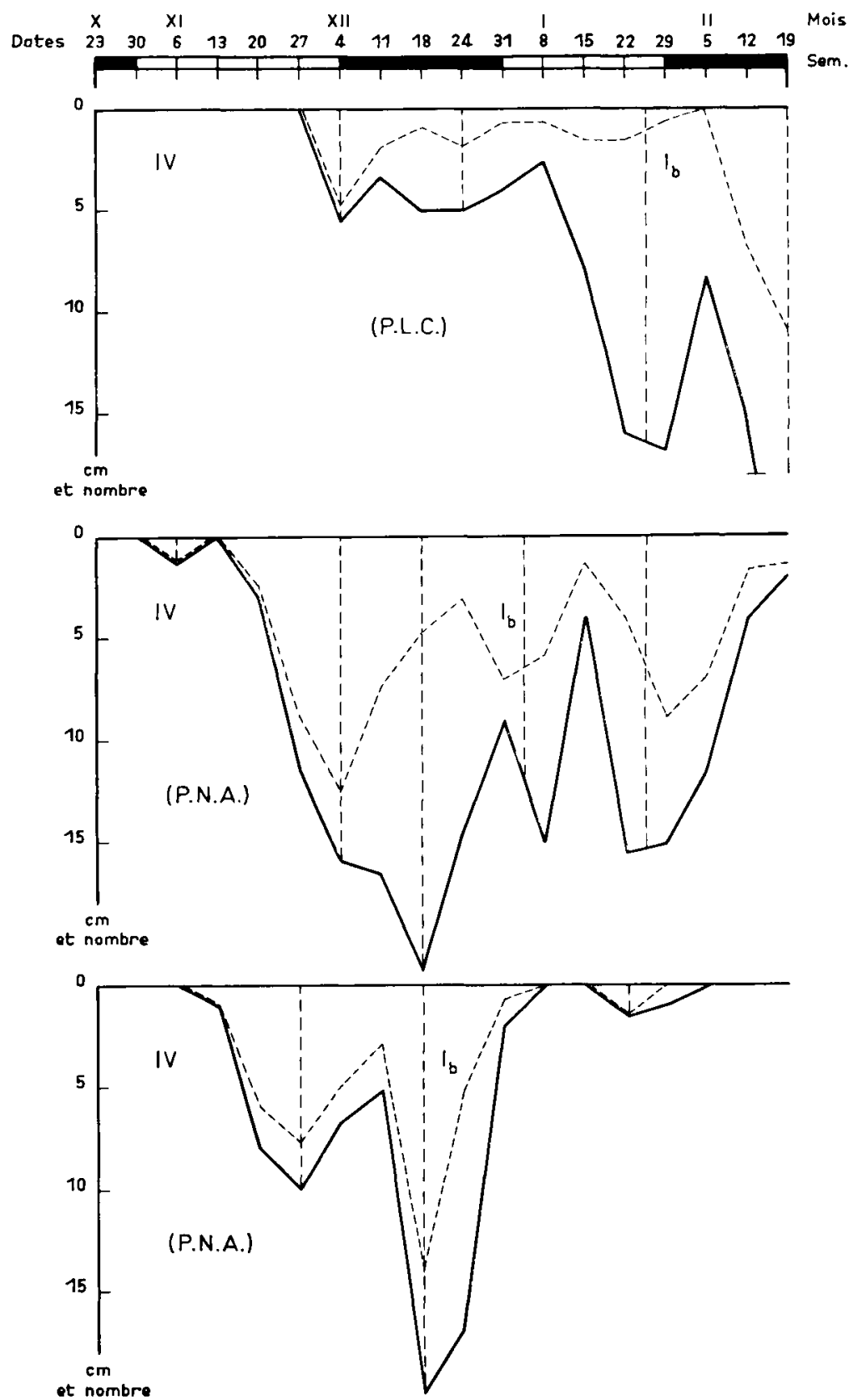

Fig. 8

Diagrammes de croissance d'un pin laricio de Corce (P.L.C.) et de deux pins noirs d'Autriche (P.N.A.) transférés avec leurs pointes blanches le 23 octobre en chambre climatisée.

Growth diagrams of Corsican Pine (P.L.C.) and Austrian Pine (P.N.A.) seedlings transfered with their white root tips on 23th October from the nursery into the growth chamber.

Notice the absence of bud break and transition from period IV to I $b$. 
Comme précédemment, à ces différentes phases se surajoutent des pics de croissance racinaire espacés de 2 à 4 semaines, particulièrement visibles pendant les phases de forte croissance racinaire. Sur les plants transférés en chambre climatisée en novembre et décembre, la phase I comporte généralement deux pics de croissance. En janvier, elle n'en comporte plus quiun.

Durant la phase III, il apparait 3 pics, dont deux généralement très forts et un troisième de moindre importance.

Ces pics, séparés par des intervalles de temps comparables aux précédents, peuvent également apparaître durant les phases II et IV, lorsque la croissance n'est pas nulle.

Sur les plants quoon a cherché à transférer en chambre climatisée avec leurs pointes blanches intactes en octobre (fig. 8), on peut observer, après la phase IV, trois pies de croissance racinaire forte avant tout débourrement.

Nous avons présenté ici surtout des diagrammes de pins laricio de Corse. Mais, ceux des pins noirs d Autriche sont strictement comparables. On notera seulement que la phase III se termine plus tard que chez le pin laricio de Corse et que la phase IV peut présenter des petits pics de croissance.

\subsection{Régénération des racines, et survie des plants transplantés en chambre climatisée et en pépinière}

\subsection{Après transfert en chambre climatisée (fig. 9)}

Pour les pins laricio de Corse, comme les pins noirs d"Autriche, le taux de survie est maximum lors du transfert des plants en décembre. A cette date, les plants provenant de la pépinière de Champenoux ne présentent pas de pointes blanches. Ce taux de survic augmente à partir d'octobre et décline après décembre, pour s'annuler quasiment vers la mi-mai. Le potentiel de régénération des racines varie de la même manière que le taux de survie. Il augmente moins vite en novembre chez le pin laricio de Corse que chez le pin noir d'Autriche, ce qui se traduit alors par un taux de survie légèrement moins élevé que pour ce dernier.

Au cours de l'été, nous avons obtenu un second pic de survie et de potentiel de régénération des racines chez le pin laricio de Corse. Chez les pins noirs d'Autriche, ce pic de ramification n'est apparu que sur les plants ayant été transférés en chambre climatisée avec leurs pointes blanches intactes.

On notera aussi que chaque fois que les plants ont pu être transférés avec leurs pointes blanches apparemment intactes, le taux de survie a généralement dépassé 70 p. 100.

\subsection{Après repiquage en pépinière (fig. 10)}

Avec les précautions prises, le taux de reprise a varié entre 70 p. 100 et 100 p. 100 . Il est proche de 100 p. 100 pour le repiquage d'automne et du début de printemps. D’avril à juillet, il décrôit jusquà 70 p. 100 . 

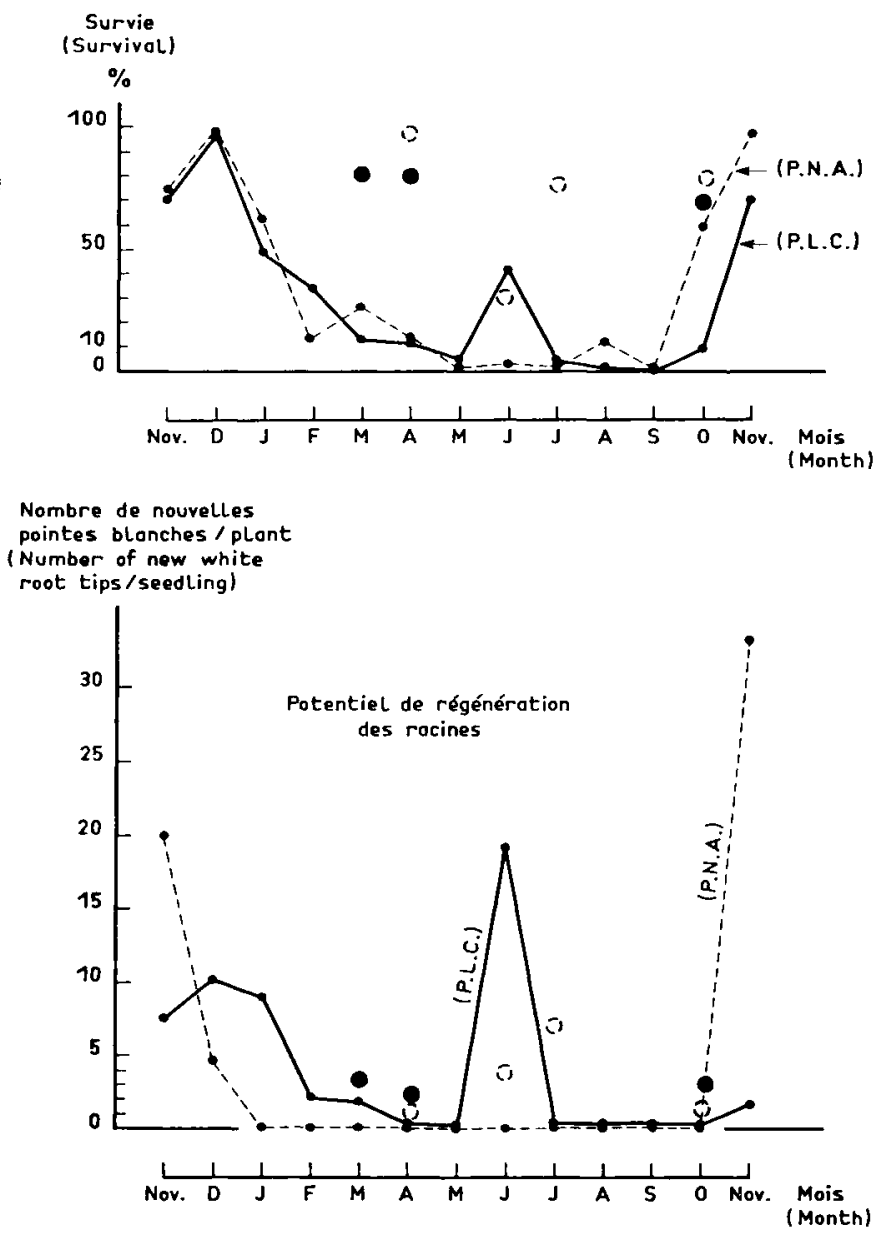

Fic. 9

Survie et potenticl de régénération de nouvelles pointes hanches sur des plants de pin laricio de Corse (- - et de pins noirs d'Autriche (- - ) transférés chaque nois en chambre climatisée.

Survival and root regeneration potential of Corsican Pine (-) and Austrian Pine (-.) seedlings transfered monthly into the growth chamber.

- Points : Plants privés de toutes leurs pointes blanches au moment du transfert.

- Cercles vides : P.N.A. dont des pointes blanches ont été conservées.

- Cercles pleins : P.L.C. dont des pointes blanches ont été conservées.

Le potentiel de régénération des racines est mesuré par le nombre de nouvelles pointes 3 semaines après le transfert en chambre climatisée.

- Points : Seedlings without any white root tips.

- Empty circles : P.N.A. on which white root tips have been kept alive.

- Full circles : P.L.C. on which white root tips have been kept alive.

The root regeneration potential is the number of new root tips appeared during the 3 weeks following the transler into the growth chamber. 

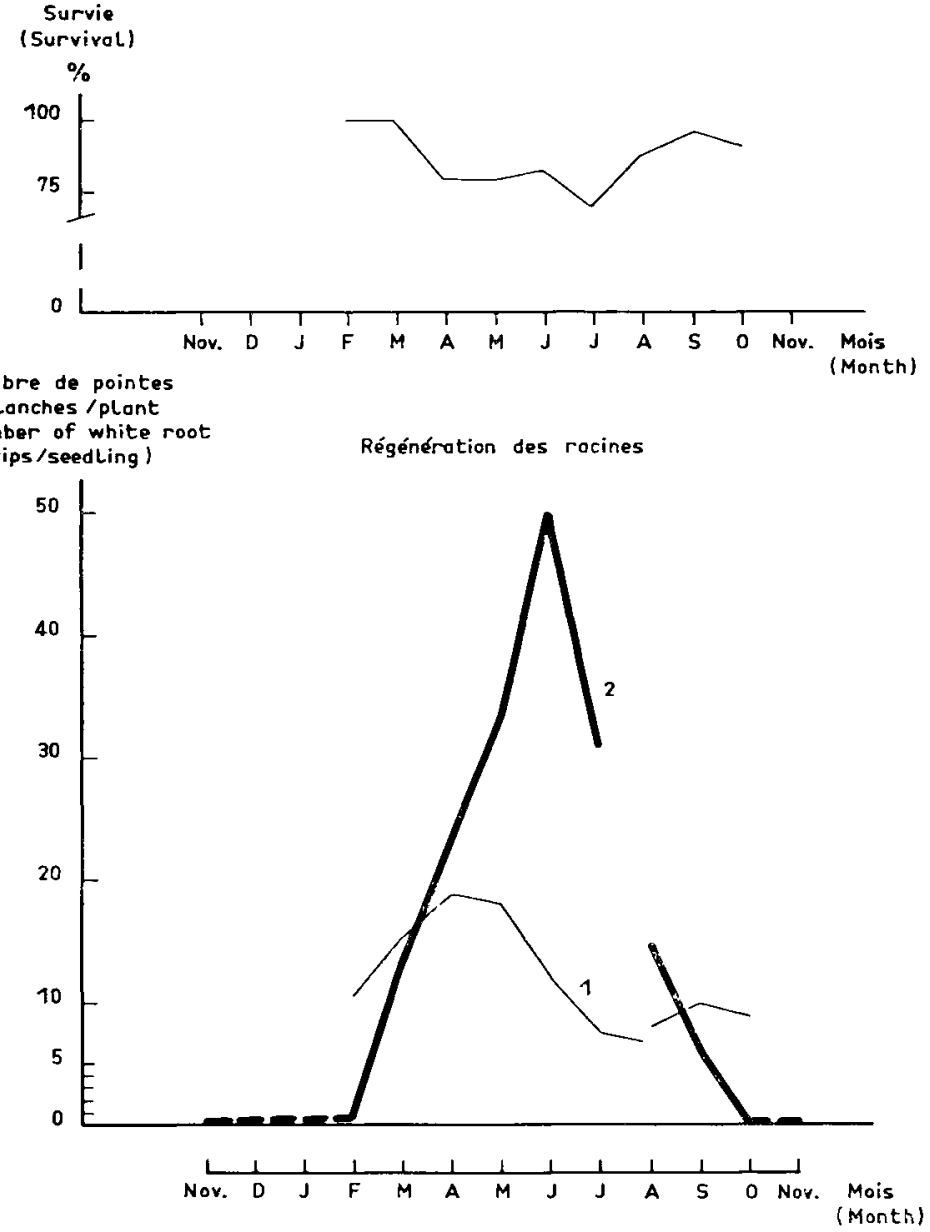

FIG. 10

Survie et régénération des racines de pins in situ pins noirs d'Autriche, pins laricio de Corse et pins laricio de Calabre confondus).

Survival and root regeneration of Pine seedlings

(Austrian, Corsican and Calabrian Pine confounded) after field transplanting.

- Haut : Survie des pins après repiquage en pépinière, sous ombrière, à différents mois de l'année à Champenoux.

- Bas : (1) Nombre moyen de pointes blanches par plant au moment du repiquage.

(2) Nombre moyen de pointes blanches par plant 4 semaines apres le repiquage.

- Top : Survival of bare root Pine seedlings transplanted in the nursery at diflerent months of the year at Champenoux.

- Bottom : (l) Mean number of white root tips at transplantation.

(2) Mean number of white root tips 4 weeks after the transplantation. 
La différence de survie entre les provenances est faible : 88 p. 100 pour le pin noir d'Autriche, 92 p. 100 pour le pin laricio de Corse et 93 p. 100 pour le pin laricio de Calabre.

En juillet, la survie du pin noir d'Autriche a été plus mauvaise que celle des deux autres sous-espèces.

La régénération des racines au cours des 4 semaines suivant la transplantation évolue en revanche différemment. De février à juin, elle augmente, tandis que d'août à décembre, elle décline. Pour des plants transplantés en pépinière, en octobre, on n’observe aucune régénération immédiate de racines. Les pins repiqués en novembre à la pépinière de Champenoux ne présentent de croissance racinaire qu'à la fin mars, comme on a pu le voir plus haut, alors que les racines des plants installés sallongent encore légèrement en octobre et en novembre.

Dans le cas présent, les accroissements sont très significativement dépendants de la date de repiquage. L'accroissement en hauteur un an après le dernier repiquage est maximum pour les arrachages d'août. De septembre à février, il reste constant, mais plus faible, puis chute brutalement de mars à mai.

\begin{tabular}{l|l|l|l|l|l|l|l|l|l|l|l|l|l|l}
\hline & $\begin{array}{l}\text { Août } \\
1970\end{array}$ & Sept. & Oct. & Nov. & Déc. & Janv. & 1971 & Fév. & Mars & Avril & Mai & Juin & Juil. \\
\hline $\begin{array}{l}\text { Accrois- } \\
\text { sement en } \\
\text { hauteur en } 1972 \\
\text { (en cm) des pins repi- } \\
\text { qués à différentes dates }\end{array}$ & 18,5 & 16,2 & 16,7 & - & - & - & 15,7 & 13,8 & 10,1 & 6,8 & 7,8 & 9,2 \\
\hline
\end{tabular}

L'évolution de l'accroissement de hauteur après repiquage varie peu avec la provenance. Tout au plus, peut-on signaler une décroissance plus rapide en mars pour le pin noir d'Autriche et une remontée encore plus spectaculaire en juillet.

\section{Conclusions et discussions}

Au cours de cette étude, il est apparu clairement, grâce à l'observation de la croissance des plants à la fois en chambre climatisée et dans les conditions naturelles, que les Pinus nigra (tant les pins noirs d’Autriche que les pins laricio de Corse) présentent 4 phases de croissance (en traits pleins sur la fig. 11), ainsi que des vagues de croissance dont les pics sont espacés de deux à quatre semaines. L'intensité de la croissance maximale des phases I et III est, de plus, modulée par la température du sol. 
En chombre climotisée
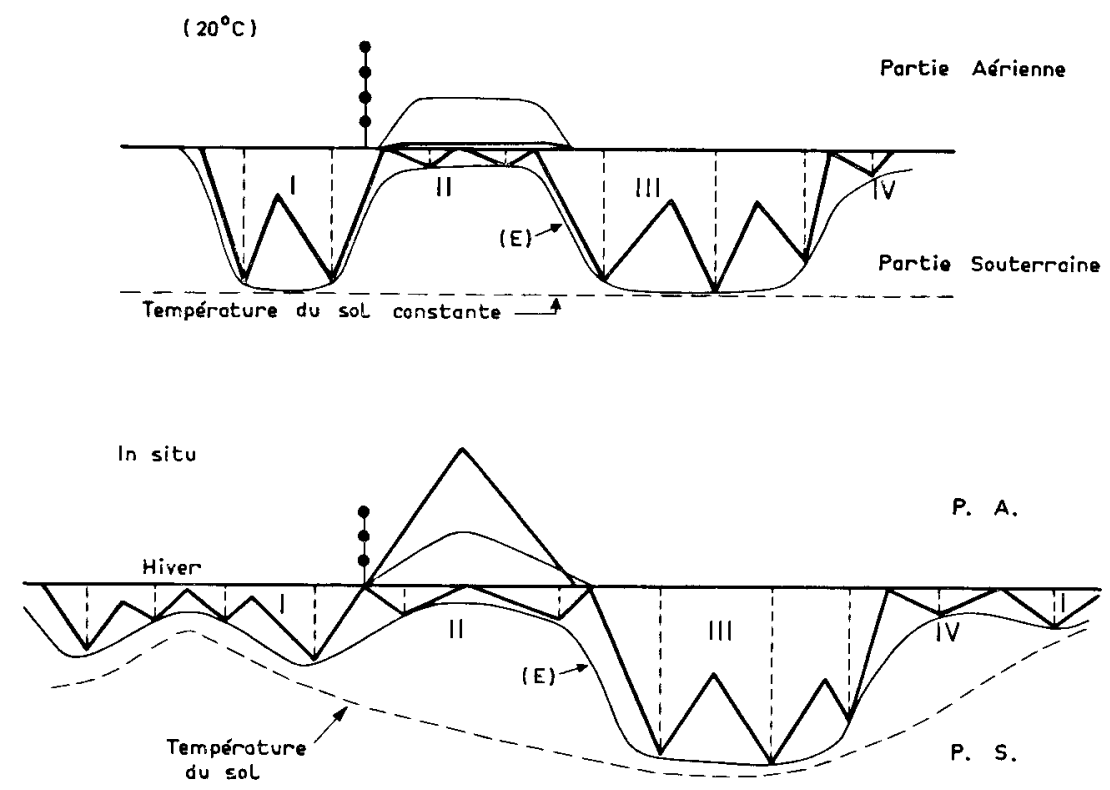

FIG. 11

Représentation schématique (en diagrammes) de la croissance d'un Pinus nigra en chambre climatisée à température constante de $20^{\circ} \mathrm{C}$ ou in situ.

Schematic growth diagrams of a Pinus nigra in the growth chamber at constant temperature or in situ.

- Partie aérienne :

- Cercle : débourrement.

- Trait fort : élongation de la pousse terminale.

- Trait fin : élongation des aiguilles.

- Partie souterraine :

- Trait fort : sommes des allongements racinaires hebdomadaires.

- Trait fin : enveloppe de la courbe des sommes des allongements racinaires hebdomadaires (E).

- Pointillé : température du sol.

- Lignes interrompues verticales : vagues de croissance.

- Aerial part:

- Circle : budburst.

- Thick line : terminal shoot elongation.

- Thin line : needle elongation.

- Subteranean part:

- Thick line : weekly extension growth of visible roots.

- Thin line : enveloppe of the weekly extension growth of visible roots.

- Dotted line : soil temperature.

- Vertical broken lines : growth waves. 
- La phase I est à croissance racinaire potentiellement forte. Dans la nature, son expression est limitée par les basses températures. Il n'y a quasiment pas d'élongation aérienne, mais les bourgeons peuvent débourrer à la fin de cette phase.

- La phase II est à croissance souterraine faible ou nulle. Elle apparaît après le débourrement, pendant la période d'élongation réelle ou potentielle des pousses aériennes.

- La phase $I I I$ est à croissance souterraine forte. Elle apparaît après la période d'élongation des pousses.

- La phase IV est à croissance souterraine faible ou nulle. Elle est également sans croissance aérienne, ce qui la distingue de la phase II.

Les vagues de croissance apparaissent régulièrement toutes les 2 à 4 semaines, sauf quand la croissance racinaire est nulle, ce qui est le cas pendant la phase $\mathbf{I}$, au cours de l'hiver pour les plants in situ sous climat très froid, et pendant la phase II en chambre climatisée. Elles sont particulièrement nettes pendant la phase III à forte intensité de croissance.

Le nombre de vagues qui peut sinscrire dans chacune des phases est fonction de leur durée. Il est beaucoup plus élevé pour la phase hivernale in situ, surtout lorsque l'hiver n'est pas trop froid (cas du schéma de la fig. 11).

Une croissance rythmique du même genre a déjà été constatée chez le cèdre (RIEDACKER, 1979). L'origine de cette rythmicité est probablement à rechercher dans le système racinaire lui-même. STREET (1967), en cultivant des racines isolées de tomate avait en effet constaté qu'elles présentaient un allongement rythmique, les intervalles entre pics de croissance étant comparables à ceux que nous avons observés. Par contre, les pics des différentes racines n'étaient pas synchronisés. Dans le cas de plantes entières, il est probable que la croissance aérienne et les variations climatiques puissent avoir un effet synchronisateur à certaines époques de l'année.

La phase II peut être repérée sans difficulté, aussi bien en chambre climatisée que dans les conditions naturelles. Elle apparaît après le débourrement, pendant la phase d'élongation des pousses aériennes, c'est-à-dire :

- en juin, pour les plants in situ; mars ;

- de fin avril à début mai, pour les plants transférés en chambre climatisée en

- en janvier, pour les plants transférés en chambre climatisée en décembre.

En chambre climatisée, l'élongation de la pousse terminale est quasiment nulle. Cela pourrait être dû a priori :

- soit à la faible intensité lumineuse régnant dans les chambres climatisées. In situ, la croissance racinaire est en effet rarement nulle, alors qu'elle l'est en chambre climatisée, notamment après le débourrement. Or, c'est à ce moment-là que la respiration est extrêmement intense et que l'assimilation nette est souvent négative chez les résineux (Bourdeau, 1959 ; NeuwirTh, 1959) ;

- soit à l'absence de basses températures nocturnes (Krugmann \& Stone, 1966). In situ pourtant, les températures nocturnes de juin sont pourtant souvent élevées;

- soit aux températures élevées que subit le système racinaire. ZanETTE (1981) a montré que l'action de températures élevées sur les racines de marcottes de pommier pouvait limiter le développement aérien. 
Une expérimentation complémentaire sera nécessaire pour déterminer les causes exactes de l'absence d'élongation des pousses terminales. Il convient toutefois de souligner que la dépression de la croissance racinaire (phase II) apparaît après le débourrement, que la pousse terminale s’allonge réellement ou non.

Lintensité de la dépression et la durée de la phase II semblent dépendre, in situ tout au moins, de la vitesse d'élongation de la partie aérienne, elle-même dépendante des conditions thermiques ambiantes.

A une croissance aérienne forte, mais peu étalée dans le temps, correspond une forte dépression de la croissance racinaire. Tout se passe comme si la partie souterraine devait alors pour sa croissance se contenter de ce dont la partic aérienne n'a pas besoin. Sur Pinus strohus, Nel.son (1964) a montré que, durant la phase d'élongation des pousses aćriennes, le transfert des glucides vers les racines cessait presque totalement.

Il est donc possible que la disponibilité en glucides utilisables joue un rôle essentiel. Mais, il n'est pas exclu non plus que des régulations hormonales limitent l'utilisation des glucides par les racines et donc leur transfert des feuilles vers les racines! La dépression racinaire a en effet lieu, même en l'absence d'élongation des pousses aćriennes.

Sur Cedrus atlantica, nous avons précédemment montré qu'en plein air comme en chambre climatisée, on obtenait une dépression analogue pendant la croissance de la partic aérienne (RIEDACKER, 1978, 1979). Comme dans le cas présent, cette croissance souterraine est davantage déprimée en chambre climatisée qu’en plein air.

Lorsque la phase 11 est retardée, la phase III à croissance racinaire forte l'est également. Celle-ci dure environ deux mois. Lorsque les plants sont repiqués une quinzaine de jours avant le début de celle-ci, leur survie peut être excellente.

Les repiquages pendant la phase III en revanche se traduisirent par des échecs. Cela nous semble pouvoir être attribué au fait que les dates de repiquage correspondaient pratiquement aux pics des vagues de croissance, de juillet et fin août, après lesquels il n'y avait que les faibles vagues de croissance du 30 août et du 3 septembre, apparemment insuffisantes pour permettre une bonne régénération des racines (fig. 7) en juillet, août.

La phase IV apparaît respectivement :

- fin août et début septembre pour les plants in situ en 1968 et 1969 ;

- en juillet pour les plants transférés en chambre climatisée en mars;

- en avril pour les plants transférés en chambre climatiscéc en décembre.

A quoi peut être attribuée cette dépression, également constatéc chez le cèdre (RiedaCKer, 1978) ? Pour le moment, nous ne pouvons quic constater son existence.

La phase I b identifiéc in situ nous paraît en réalité être le début de la phase I (fig. 3 et 4). Selon les cas, elle peut durer plus ou moins longtemps. Lorsque les plants sont aptes à débourrer avant la fin de la phase IV, comme cela a pu se produire pour des plants transférés en chambre climatisée, en mars ou en décembre, il semble même qu'elle puisse disparaître. Plus généralement cependant, elle peut dans ces conditions apparaître, mais de façon brève, lorsque le débourrement et l'allongement 
aérien peuvent se produire rapidement. Elle peut durer plus longtemps et comporter deux ou trois vagues de croissance lorsque les plants sont entrés normalement en dormance avant leur transfert en chambre climatisée en octobre (fig. 8). Elle peut enfin durer jusqu'en mai pour des plants en conditions naturelles. Elle peut cependant être entrecoupée par un arrêt total de la croissance racinaire de fin novembre à fin mars à Champenoux, sous l'effet de températures ambiantes trop basses.

En effet, Palashev \& Monalova (1973) ont pu observer que des pins noirs situés dans la partie la plus chaude du Sud-Est de la Bulgarie présentaient une croissance racinaire faible, mais non nulle, durant tout l'hiver. A plus forte altitude, c'està-dire à plus basses températures, celle-ci s'annulait en décembre et en janvier.

Les pins transférés en chambre climatisée en décembre et janvier peuvent d'ailleurs régénérer des racines qui pourront immédiatement s'allonger. Contrairement à ce qui se passe en octobre, les plants transférés en novembre, décembre et janvier, peuvent débourrer rapidement. Tout se passe alors comme si une nouvelle séquence comportant les 4 phases pouvait alors se dérouler normalement. La vitesse de déroulement de ces phases semble alors dépendre essentiellement de la température aérienne.

A basse température, elle paraît retardée. In situ, la phase I dure jusqu'en mai. Mais, lorsque les plants sont transférés en chambre climatisée à $20^{\circ} \mathrm{C}(16 \mathrm{~h}$ de jour $)$ avec leurs pointes blanches, cette phase n'est observable que jusqu'en mars. Et si, de surcroît, les pointes blanches ont été supprimées, elle n'est plus observable dès le mois de février. Pendant la régénération des racines, qui nécessite en effet en général une quinzaine de jours à $20^{\circ} \mathrm{C}$, la plante entre dans la phase II grâce aux températures aériennes élevées. Or, cette deuxième phase, à faible croissance racinaire, est aussi à faible possibilité de régénération de racines. Elle est extrêmement défavorable pour la survie des plants.

\section{Les températures ambiantes conditionnent donc deux phénomènes :}

- d'une part, la température du sol conditionne l'expression des potentialités de croissance. En effet, la croissance hebdomadaire maximale durant la phase I est aussi importante en chambre climatisée que durant la phase III, mais, in situ, elle est fortement diminuée et peut même s'annuler par suite des basses températures ;

- d'autre part, la température de l'air conditionne la vitesse de passage d'une phase à une autre.

Au printemps, lorsque laair se réchauffe, et donc aussi le sol, les possibilités de croissance, de régénération et de croissance des racines augmentent. Mais, simultanément, le plant passe de la phase I à la phase II, ce qui produit l'effet inverse. Si cette interprétation est exacte, le réchauffement du sol et le maintien de la partie aérienne à une température relativement basse, mais compatible avec une photosynthèse correcte, devraient se traduire par une croissance et une régénération des racines importantes. C'est ce que nous nous proposons de vérifier.

Ces considérations permettent de suggérer des conseils pour les praticiens et des voies nouvelles de recherche. Pour le reboisement de printemps, il conviendrait sans doute de localiser la production de plants dans des pépinières à printemps frais, de façon à ralentir le passage à la phase II qui est défavorable à la régénération et à la croissance des racines. La conservation hivernale des plants en chambre froide devrait également retarder le passage à la phase II. 
Pour des reboisements d'automne, il conviendrait au contraire de hâter l'arrivée de la phase I, donc d'accélérer le déroulement des phases précédentes, afin de pouvoir planter tant que le sol est encore suffisamment chaud. Ce résultat devrait pouvoir être atteint en localisant la pépinière dans un climat précocement chaud ou en élevant la température à laaide d'un tunnel en plastique.

Il conviendrait de préciser les divers moyens de décaler les phases. Une sécheresse de juin ou juillet peut-elle modifier la durée et l'apparition des phases II, III, IV et I ? On sait en effet qu'une sécheresse d'été peut, chez le cèdre, provoquer un débourrement plus précoce au cours de l'année suivante (Finkelstein, 1981). Il n'est donc pas impossible qu'elle puisse aussi hâter l'entrée dans les périodes III, II et I et entraîner de ce fait une croissance racinaire d'automne plus importante.

La phase III, à forte potentialité de croissance, pourrait être utilisée pour les repiquages d'été de juillet ou d'août selon les années. Il conviendrait pour cela d'arracher les plants lorsque l'élongation des pousses a cessé. Certains pépiniéristes mettent cette potentialité à profit et repiquent effectivement leurs plants avec succès en été.

On pourrait également envisager de soulever les plants à cette date. Le reboisement de plants en mottes (avec les pointes racinaires blanches intactes) à la fin de lété, pourvu que le sol soit suffisamment humide, devrait également permettre d’obtenir la meilleure installation des plants; la croissance racinaire est alors potentiellement forte, et c'est ainsi quion obtient la plus forte élongation de la pousse terminale au cours de l'année suivante. Un repiquage sur le terrain de fin d'été de plants à racines nues, théoriquement possible, paraît devoir donner des résultats plus aléatoires.

En automne, les plants à pointes blanches présentent encore une légère supériorité par rapport aux plants à racines nues. Ces derniers, lorsqu'ils sont repiqués en octobre, ne régénèrent pas de racines avant le printemps, alors que les plants en place ont des racines dont l'allongement se poursuit en octobre et même en novembre.

Un repiquage pendant la période II a pour effet de diminuer au maximum l'élongation de la pousse terminale de lannée. Plus la plantation ou le repiquage en pépinière ont lieu tardivement au printemps, plus la croissance de la pousse terminale est réduite. Et, c'est en mai, en pleine phase II, que cette dépression est maximale.

Dans la présente étude, le pin laricio de Corse transplanté in situ ou en chambre climatisée ne résiste pas moins bien que le pin noir d'Autriche.

Le potentiel de régénération des racines des deux sous-espèces évolue d'ailleurs presque parallèlement. S'il y a réellement des différences d’aptitude à la survie après transplantation, comme le prétendent les praticiens, l'explication est peut-être à rechercher plutôt dans la capacité de résistance à la sécheresse des deux sous-espèces, lorsquelles sont privées de pointes racinaires blanches. Aussenac (1980) a en effet pu observer que les rameaux de pins noirs d'Autriche qui seraient en réalité généralement originaires de Croatie, ont de meilleures capacités de régulation stomatiques que ceux de pin laricio de Corse.

De nombreux auteurs utilisent, depuis Stone \& Schubert (1959), avec plus ou moins de succès, la mesure du potentiel de régénération des racines (PRR) pour déterminer les chances de survie après transplantation de plants arrachés à différents mois de l'année. Ritchie \& Dunlap (1980) constatent, au cours de leur revue biblio- 
graphique, qu’il y a en général chez les résineux une assez bonne corrélation entre l'aptitude à la survie après transplantation in situ et l'intensité du potentiel de régénération des racines.

Mais que signifie cette mesure ? Les auteurs qui l'ont utilisée n'en n'ont pas donné d'interprétation. A la lumière du présent travail, il nous semble possible de donner la signification et les limites de ce test dit de STONE (1959). Quand le potentiel de régénération des racines en automne est bas, c'est que le plant est encore dans la phase IV. Lorsqu'il augmente, c'est que le plant entre ou est entré dans la phase I. Enfin, lorsqu'il revient à un niveau très bas, c'est que les plants sont dans la phase II, ou sur le point d'y entrer.

Comme nous l'avons suggéré plus haut, la vitesse d'évolution du plant paraît en effet dépendre de la température à laquelle est soumise la partie aérienne. A cela, se surajoutent les vagues de croissance qui, d'une semaine à l'autre, lorsqu'elles sont bien synchronisées, peuvent faire varier fortement la valeur du potentiel de régénération des racines.

La régénération des racines dépend donc à la fois de la température du sol, qui permet l'expression du potentiel de régénération, et de la température de l'air qui, dans les tests, est généralement bien supérieure à ce qu'elle est dans les conditions naturelles. Dans nos expériences, la température était en permanence de $20^{\circ} \mathrm{C}$, et nous avons obtenu le maximum de potentiel de régénération des racines en décembre pour des pins laricio de Corse, ainsi que pour des pins noirs d'Autriche élevés à Champenoux.

Franclet \& Poissonnier (1974) ont, pour du pin laricio de Calabre élevé en Sologne et testé dans un climat un peu plus frais $\left(20^{\circ} \mathrm{C}\right.$ le jour, $18{ }^{\circ} \mathrm{C}$ la nuit) obtenu un maximum en janvier. Il est à prévoir que plus la température de l'air au printemps sera basse, plus l'entrée dans la phase II sera retardée, et plus le potentiel de régénération des racines sera élevé pendant une période plus Iongue. Dans ces conditions, à un stade donné, un plant repiqué dans un sol chaud, pendant une période encore fraîche, aura plus de chance de survivre qu'un plant planté dans un sol chaud, mais avec une température de l'air élevée. Un brusque et important réchauffement de l'air sur un sol froid, faisant passer rapidement le plant au stade II, sera le cas le plus défavorable pour une bonne installation du plant. Ces dernières remarques permettent de comprendre pourquoi la mesure standard du potentiel de régénération des racines ne peut rendre compte qu'imparfaitement des chances de survie d'un plant venant d'être repiqué.

Reçu pour publication en avril 1982.

\section{Summary \\ Root growth and root regeneration of Corsican and Austrian pine seedlings in growth chambers and in natural conditions}

We recorded root growth and regeneration on one hand and shoot and needle growth on the other hand, on young Austrian Pine ( $P$. nigra ssp nigricans Host.) and Corsican Pine (P. nigra ssp laricio Poir.) : 
1) grown either in minirhizotrons in growth chambers $120^{\circ} \mathrm{C}-16 \mathrm{~h}$ light 2500 to 3000 Lux) for about 6 months or outdoor's behind a root cellar with a transparent side, cluring 2 years :

2) taken out each month from the nursery and transplanted either in the nursery or the growth chamber in minirhizotrons.

Four root growth periods were found : Period I preceeding bud burst, with a high root growth potential, but which expression in situ is limited by temperature; Period il with a low root growth potential and which takes place during shoot elongation: a long Period III, with high root growth potential which begins at the end of the shoot clongation : a Period IV, with low shoot growth potentiality which takes place at the end of the summer. Duration of the different periods depends on shoot tenuperature, whereas the expression of root growth and root regeneration potentialities depends on soil temperature. Outdoors, root growth may stop completely due to low soil temperatures.

Within these growth periods appear growth waves every $3 \pm 1$ weeks. These waves are particularly evident during period $\mathrm{III}$ in growth chambers as well as outdoors. White root tips, even a few, secm to help seedling to remain alive for some time, often until a new and strong root growth wave appears. The root regeneration potential concept is reinterpreted and its validity discussed.

From a practical point of view nursery transplantation in late summer, at the beginning of period III, just after the end of shoot elongation, allows the best shoot growth during the following year, whereas transplantation during period II particularly in March, although possible in the nursery depresses drastically shoot growth. Undercutting should therefore also induce the least shoot growth depression when praticed at the beginning of period III. Summer transplantation of container grown seedling should also as sometimes stated, allow a good soil colonization and the weakest transplantation shock.

Due to the effect of temperature on the duration of the dilferent growth periods, reforestation with bare root seedlings should probably be carried out in autumn and in spring with seedlings coming from nurseries with different climatic conditions.

\section{Références bibliographiques}

Arbez M., 1971. Croissance des racines du pin laricio de Corse (Pinus nigra ARN ssp laricio) au stade juvénile. Ann. Sci. for., 28 (3), 259-288.

Aussenac G., 1980. Comportement hydrique de rameaux excisés de quelques espèces de sapins et de pins noirs en phase de dessication. Amn. Sci. for., 57 (3), 201-215.

Bourdeau Ph.F., 1959. Seasonal variations of the photosynthetic efficiency of evergreen conifers. Ecology, 40, 63-67.

Coic Y., Lesaint C., 1973. La nutrition minérale en horticulture. Revue horticole, aoûtsept., 2, 316.

FinKelstein D., 1981. Influence des conditions d'alimentation hydrique sur le débourrement et la croissance de jeunes plants de cèdres (Cedrus atlantica Manetti) cultivés en serre. Ann. Sci. for., 38 (4), 513-530.

Franclet A., Poissonnier M., 1975. Le test de Stone permet d'apprécier l'aptitude physiologique des plants forestiers à surmonter la crise de transplantation. In : Compte rendu des Séminaires du Groupe d'Etude des Racines, tome 2, 1975, 146-161. Edité par J. Gagnaire-Michard et A. Riedacker, I.N.R.A., F 54280 Seichamps.

Krugmann S.L., Stone E.C., 1966. The effect of cold nights on the root regenerating potential of Ponderosal pine seedlings. For. Sci., 12, $451-459$.

NELSON C.D., 1964. The production and translocation of photosynthate $C^{11}$ in conifers. The Formation of Wood in Forest Trees. Symposium held at Harvard. Edit. by M.H. Zimmermann, Academic Press, 243-257.

Neuwirti G., 1959. Der $\mathrm{CO}_{2}$ Stoffwechsel einiger Coniferen whärend des Knospenaustriebes. Biol. $Z$ bl., 78, 559-584. 
Palashev I., Monolova, 1974. Periodicity and intensity of root growth of the trees of Pinus maritima Poir. and Pinus nigra arn in S.E. of Bulgaria. II. - International Symposium Ecology and Physiology of root growth. A kademie Verlag, Berling, 309-315.

Riedackir A., 1974. Un nouvel outil pour l'étude des racines et de la rhizosphère : le minirhizotron. Ann. Sci. for., 31 (2), 129-134.

Rifodcker A., 1976. Rythmes de croissance et de régénération des racines des végétaux ligneux. Ann. Sci. for., 33 (3), 109-138.

Riedacki:R A., 1978. Régénération et croissance de la partie souterraine et aérienne de cèdres placés sous climat constant. Ann. Sci. for., 35 (2), 117-138.

RIIDACKliR A., 1979. Growth and regeneration rythms of Cedrus atlantica roots in situ and in constant climate. In Comptes rendus du Symposium "Physiologie des racines et symbioses » du groupe I.U.F.R.O. S2.01-13 et du Groupe Français d'Etude des Racines, Nancy, 11-15 septembre 1978. Riedacker Editeur, 71-85.

RitchiE G.A., Duni.AP J.R., 1980. Root growth potential : its development and expression in forest tree seedlings. New-Zealand, J. for. Sci, 10 (1), Special issue Planting stock quality, $218-248$.

Stone E.C., Schubert G.H., 1959. Root regeneration by ponderosa pine seedlings lifted at different times of the year. For. $S_{c i}$, 5, 322-332.

Streer H.E., 1967. The ageing of root meristems. Aspect of the biology of ageing. Symposia of the Society of experimental biology, 517-542.

ZANeTTE F., 1981. Recherches descriptives et expérimentales sur la morphogénèse de systèmes aériens et racinaires de quelques porte-greffes de pommiers. Thèse Université de Clermont-Ferrand II. 